\title{
Topological Properties of the Immediate Basins of Attraction for the Secant Method
}

\author{
Laura Gardini, Antonio Garijo and Xavier Jarquee
}

\begin{abstract}
We study the discrete dynamical system defined on a subset of $R^{2}$ given by the iterates of the secant method applied to a real polynomial $p$. Each simple real root $\alpha$ of $p$ has associated its basin of attraction $\mathcal{A}(\alpha)$ formed by the set of points converging towards the fixed point $(\alpha, \alpha)$ of $S$. We denote by $\mathcal{A}^{*}(\alpha)$ its immediate basin of attraction, that is, the connected component of $\mathcal{A}(\alpha)$ which contains $(\alpha, \alpha)$. We focus on some topological properties of $\mathcal{A}^{*}(\alpha)$, when $\alpha$ is an internal real root of $p$. More precisely, we show the existence of a 4-cycle in $\partial \mathcal{A}^{*}(\alpha)$ and we give conditions on $p$ to guarantee the simple connectivity of $\mathcal{A}^{*}(\alpha)$.
\end{abstract}

Mathematics Subject Classification. 37G35, 37N30, 37C70.

Keywords. Root finding algorithms, rational iteration, secant method, periodic orbits.

\section{Introduction and Statement of the Results}

Dynamical systems is a powerful tool to have a deep understanding on the global behavior of the so called root-finding algorithms, that is, iterative methods capable to numerically determine the solutions of the equation $f(x)=0$. In most cases, it is well known the order of convergence of those methods near the zeros of $f$, but it is in general unclear the behavior and effectiveness when initial conditions are chosen on the whole space; a natural question when we do not know a priori where the roots are or if there are many of them.

The numerical exploration of the solutions of the equation $f(x)=0$ has been always central problem in many areas of applied mathematics, from biology to engineering, since most mathematical models require to have a thorough knowledge of the solutions of certain equations. Once we are certain that no algebraic manipulation of the equation will allow to explicitly find

This work has been partially supported by MINECO-AEI grants MTM-2017-86795-C3-2-P and MTM-2017-86795-C3-3-P and the AGAUR grant 2017 SGR 1374. 
out the solutions, one can try to built numerical methods which will approximate the solutions with arbitrary precision. Perhaps, the most well-known and universal method is the Newton method inspired on the linearization of the equation $f(x)=0$. But also other methods have shown to be certainly efficient like the secant method, the main object of the paper.

Roughly speaking, all these iterative methods give efficient ways to find the solutions of $f(x)=0$, at least once you have a good approximation of them. However, there is a significant amount of uncertainty when the initial conditions are freely chosen, i.e., when there is not a natural candidate for the solution or the number of solutions is high. It is in this context where dynamical systems might play a central role. As an example we can refer to [7] where the authors first prove theoretical results on the global dynamics of the Newton method and then apply them to create efficient algorithms to find out all solutions, even in the case that the degree of $p$ is huge.

This paper is a step forward in this direction for the secant method. This method has some advantages over Newton's method, but the dimension of the natural phase space of the associated iterative system changes from 1 to 2. Correspondingly, its study requires new techniques and ideas. See also $[1,5]$.

Let $p$ be a real polynomial of degree $k$ given by

$$
p(x)=a_{0}+a_{1} x+\text { cdots }+a_{k} x^{k} \text { with } \quad a_{k} \neq 0 .
$$

We assume that $p$ has exactly $n \in\{3, \ldots k\}$ simple real roots denoted by $\alpha_{0}<\alpha_{1}<\cdots<\alpha_{n-2}<\alpha_{n-1}$. Accordingly if $p$ has other roots different from $\alpha_{j}, j=0, \ldots n-1$, they are non-real complex conjugate pairs with no further assumptions on them. On the other hand, the case of multiple real roots of $p$ has been partially studied in [6]. We named the real roots of $p$ as follows. The roots $\alpha_{0}$ and $\alpha_{n-1}$ (smallest and biggest) are called the external roots of $p$; in contrast the other real roots of $p$, i.e., $\alpha_{j}$ with $j \in\{1, \ldots, n-2\}$, are called the internal roots of $p$.

We consider the secant method applied to the polynomial $p$ as a discrete dynamical system acting on the real plane,

$$
S:=S_{p}: \mathbb{R}^{2} \mapsto \mathbb{R}^{2}, \quad S:\left(\begin{array}{l}
x \\
y
\end{array}\right) \mapsto\left(\begin{array}{l}
y \\
y-p(y) \frac{y-x}{p(y)-p(x)}
\end{array}\right),
$$

and the orbit of the seed $\left(x_{0}, y_{0}\right) \in \mathbb{R}^{2}$ is given by the iterates of the map; that is, the sequence $\left\{S^{m}\left(x_{0}, y_{0}\right)\right\}_{m \geq 0}$. We refer to [5] for a detailed discussion of the two-dimensional dynamical system induced by $S$ and also some consequences as a root finding algorithm. Here, we will always consider $S: \mathbb{R}^{2} \rightarrow \mathbb{R}^{2}$, but there is a natural extension of this problem by assuming $p$ as a polynomial with complex coefficients and thus, $S: \mathbb{C}^{2} \rightarrow \mathbb{C}^{2}$. See [1] for a discussion on this context.

Any simple root $\alpha$ of $p$ corresponds to an attracting fixed point $(\alpha, \alpha)$ of the secant map $S$. Thus, we can consider the basin of attraction of $(\alpha, \alpha)$, denoted by $\mathcal{A}(\alpha)$, consisting of all points tending towards this fixed point,

$$
\mathcal{A}(\alpha)=\left\{(x, y) \in \mathbb{R}^{2} ; S^{m}(x, y) \rightarrow(\alpha, \alpha) \text { as } m \rightarrow \infty\right\} .
$$




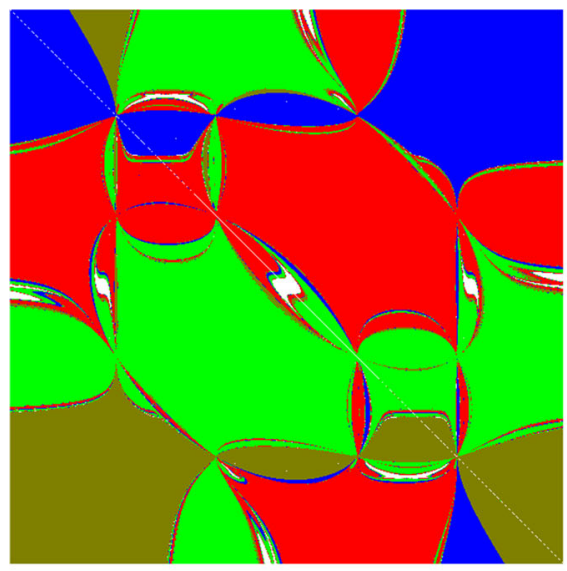

(a) $T_{4}(x)=8 x^{4}-8 x^{2}+1$.

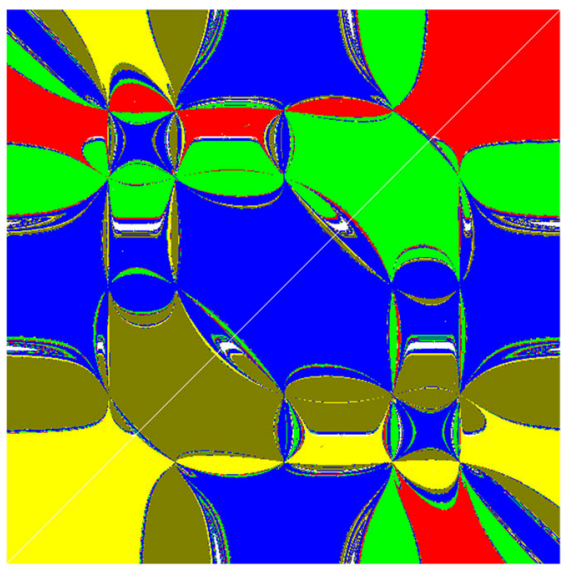

(b) $T_{5}(x)=16 x^{5}-20 x^{3}+5 x$.

Figure 1. Phase plane of the secant map applied to the Chebyshev polynomials $T_{k}(x)$ for $k=4$ and 5 . We show each basin of attraction with a different color. Range of the pictures $[-1.5,1.5] \times[-1.5,1.5]$ (color figure online)

It is easy to see that when $\alpha$ is a simple root of $p$ then the point $(\alpha, \alpha)$ belongs to $\operatorname{Int}(\mathcal{A}(\alpha))$. However this is not always the case when $\alpha$ is a multiple root of $p$ (see [6]). It is remarkable that even in the case of $\alpha$ being a simple root of $p$ the local dynamics around the point $(\alpha, \alpha)$ does not follow the typical behavior of an attracting fixed point of a diffeomorphism ( $\grave{a}$ la HartmanGrobman) due to the presence of infinitely many points, in any neighborhood of the fixed point, which under one iteration land on the fixed point.

We also denote by $\mathcal{A}^{*}(\alpha)$ the immediate basin of attraction of $(\alpha, \alpha)$, i.e., the maximal connected component of $\mathcal{A}(\alpha)$ containing $(\alpha, \alpha)$. Moreover, if $\alpha$ is an external root of $p$ then its immediate basin of attraction is an unbounded set while if $\alpha$ is an internal root then $\mathcal{A}^{*}(\alpha)$ is bounded (See [5]). This property shows the first difference between the immediate basin of attraction of an external and an internal simple root.

Along the paper, we use Chebyshev polynomials $T_{k}(x)$ for $k \geq 0$ for computer pictures to illustrate our results. We recall that Chebyshev polynomials can be defined by $T_{0}(x)=1, T_{1}(x)=x$ and recursively $T_{k+1}(x)=$ $2 x T_{k}(x)-T_{k-1}(x)$ for $k \geq 1$. Among other properties every polynomial $T_{k}(x)$ has degree $k$ and exhibits $k$ simple real roots in the interval $(-1,1)$. Indeed the roots of $T_{k}$ are located at points $x_{j}=\cos \left(\frac{\pi(j+1 / 2)}{n}\right)$, for $j=0, \ldots, k-1$. In Fig. 1 we show the phase plane of the Secant maps for the polynomials $T_{k}$ for $k=4$ and 5 , and in Fig. 10a for $k=3$. The range of the picture is $[-1.5,1.5] \times[-1.5,1.5]$ so the points $(\alpha, \alpha)$ are located at the diagonal of each picture. The topological structure of the immediate basin of attraction seems to remain similar depending only on the character of the root (internal or 

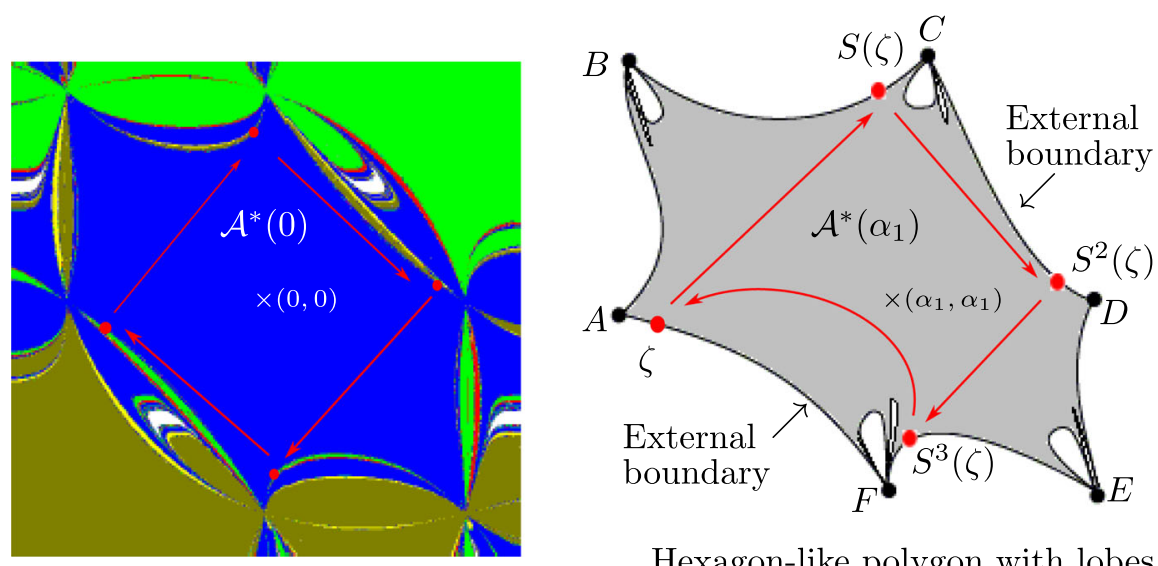

Hexagon-like polygon with lobes

Figure 2. On the left-hand side, we show the phase space of the secant map applied to the Chebyshev polynomial $T_{5}(x)=16 x^{5}-20 x^{3}+5 x$ (see Fig. 1c), we show in blue $\mathcal{A}^{*}(0)$. Range of the phase plane $[-0.75,0.75] \times[-0.75$, $0.75]$. On the right-hand side, we sketch an hexagon-like polygon with lobes which is the topological model of the immediate basin of attraction of an internal root $\alpha_{1}$. The six vertices $A, B, C, D, E$ and $F$ of the hexagon are focal points and we only plot two (of countable many) lobes attached to the focal points $B, C, E$ and $F$. We also sketch the presence of a 4 -cycle $\zeta, S(\zeta), S^{2}(\zeta), S^{3}(\zeta)$ lying on the external boundary in red color (color figure online)

external). To state the main results on this direction, we first introduce some required notation.

Let $T \subset \mathbb{R}^{2}$ be a bounded (infinite) graph formed by vertices and edges. We say that an edge of $T$ is a lobe if it connects a vertex with itself. We say that $T$ is a smooth hexagon-like polygon with lobes if it is formed by six vertices, six $C^{1}$-edges connecting those vertices and countably many $C^{1}$-lobes at some of the vertices.

The main goal of this paper is to describe the topology of the immediate basin of attraction of an internal root of $p$. In Fig. 2 (left), we plot the immediate basin of attraction $\mathcal{A}^{*}(0)$ for the Chebyshev polynomial $T_{5}(x)=16 x^{5}-20 x^{3}+5 x$ while in Fig. 2 (right) we give the skeleton of the boundary of the immediate basin of attraction $\mathcal{A}^{*}(\alpha)$ of an internal root. There we illustrate the smooth hexagon-like polygon with vertices at the points $A, B, C, D, E$ and $F$. This hexagon-like polygon coincides with the external boundary of $\mathcal{A}^{*}(\alpha)$. But the boundary of an immediate basin also contains some (infinitely many) lobes attached to the vertices $B, C, E$ and $F$ (as illustrated on the picture); those lobes (except the vertices) are part of $\partial \mathcal{A}^{*}(\alpha)$ but not part of the external boundary. We collect the main results on two statements. The first one is about the topology of the external boundary 
of $\partial \mathcal{A}^{*}(\alpha)$ and its dynamics. Focal points and lobes will be defined in Sect. 2 . See Fig. 2.

Theorem A. Let $\alpha_{1}$ be an internal root of $p$ and let $\alpha_{0}<\alpha_{1}<\alpha_{2}$ be simple consecutive roots of $p$. The following statements hold, provided the external boundary is piecewise smooth.

(a) $\partial \mathcal{A}^{*}\left(\alpha_{1}\right)$ contains an hexagon-like polygon with lobes where the vertices are the focal points $Q_{i, j} i \neq j \in\{0,1,2\}$.

(b) There exists a 4-cycle in $\partial \mathcal{A}^{*}\left(\alpha_{1}\right)$.

Second, we investigate the connectedness of the immediate basin of attraction. Looking at the examples in Fig. 1 the immediate basin of attraction of an internal root seems to be simply connected. However, it is easy to find examples where $\mathcal{A}^{*}(\alpha)$ is multiply connected. See Fig. 3 . In the next result, we find sufficient conditions to assure that the immediate basin of attraction of an internal root is a simply connected set.

Theorem B. Let $\alpha_{1}$ be an internal root of $p$ and let $\alpha_{0}<\alpha_{1}<\alpha_{2}$ be simple consecutive roots of $p$ such that $p$ has only one inflection point in the interval $\left(\alpha_{0}, \alpha_{2}\right)$. If the external boundary of $\mathcal{A}^{*}\left(\alpha_{1}\right)$ is piecewise smooth, then it is simply connected.

From Theorems A and B, we can conclude the following corollary that applies to any real polynomial of degree $k$ with exactly $k$ simple real roots, as the family of Chebyshev polynomials.

Corollary C. Let $p$ be a polynomial of degree $k$ with exactly $k$ simple real roots and one, and only one, inflection point between any three consecutive roots of $p$. Then for any internal root $\alpha$ of $p$, the immediate basin of attraction, $\mathcal{A}^{*}(\alpha)$, is a simply connected set and $\partial \mathcal{A}^{*}(\alpha)$ is an hexagon-like polygon with lobes where the vertices are focal points. Moreover, there exists a 4-cycle in $\partial \mathcal{A}^{*}(\alpha)$.

The paper is organized as follows. In Sect. 2, we introduce the terminology and tools on rational iteration on the plane. In Sect. 3, we classify the cycles of minimal period 4 of the secant map. In Sects. 4 and 5 , we prove Theorems A and B, respectively.

\section{Plane Rational Iteration}

For the sake of completeness, we briefly summarize the notions, tools and results from [2-4] which are needed here. Consider the plane rational map given by

$$
T:\left(\begin{array}{l}
x \\
y
\end{array}\right) \mapsto\left(\begin{array}{l}
F(x, y) \\
N(x, y) / D(x, y)
\end{array}\right),
$$

where $F, N$ and $D$ are differentiable functions. Set

$$
\delta_{T}=\left\{(x, y) \in \mathbb{R}^{2} \mid D(x, y)=0\right\} \quad \text { and } \quad E_{T}=\mathbb{R}^{2} \backslash \bigcup_{n \geq 0} T^{-n}\left(\delta_{T}\right) .
$$




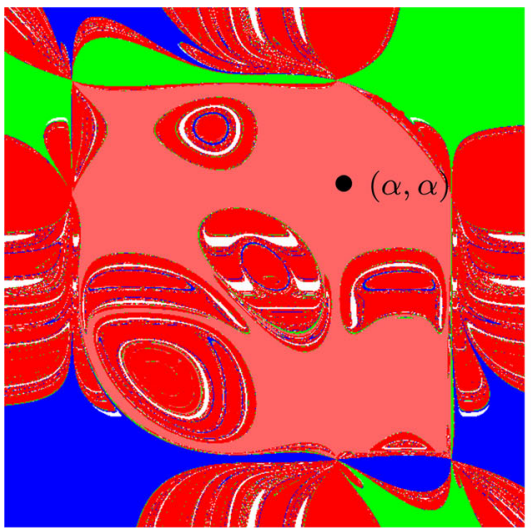

(a) $p_{1}(x)=16 x^{5}-20 x^{3}+x+0.8$.

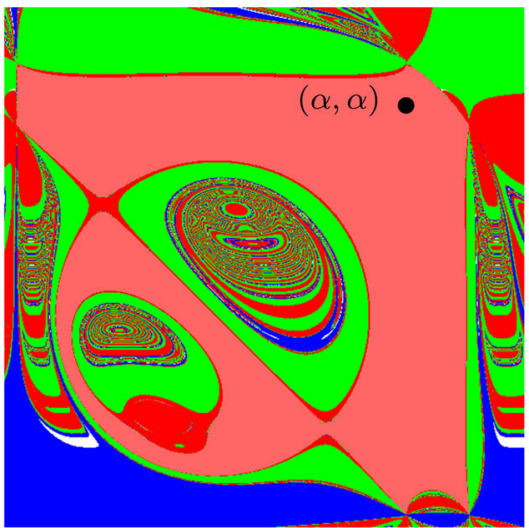

(b) $p_{2}(x)=\frac{x 5}{5}-\frac{x^{3}}{3}-0.05 x+0.15$.

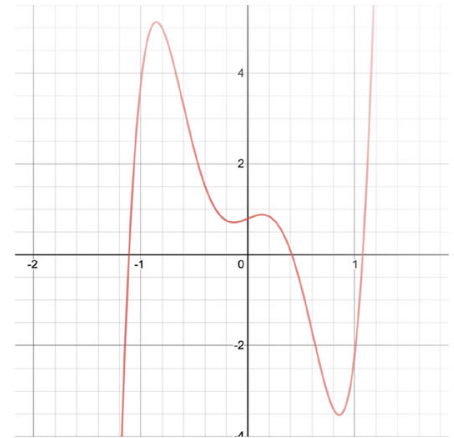

(c) Graph of the polynomilal

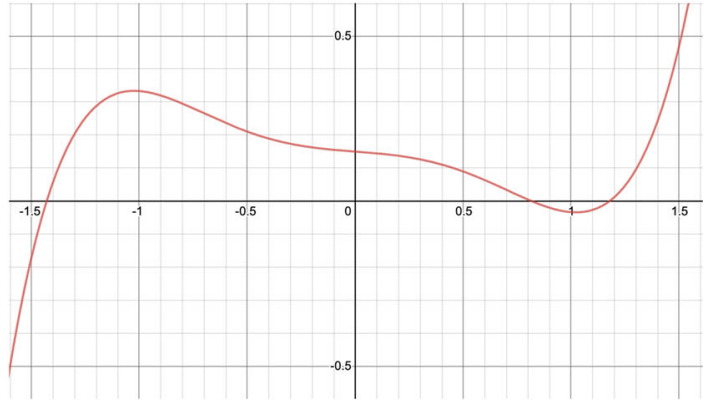

(d) Graph of the polynomial $p_{2}$. $p_{1}$.

Figure 3. Phase plane of the secant map applied to two degree five polynomials (with only tree real roots) where the immediate basin of attraction of an internal root is multiply connected. In both cases, color blue and green refer to the attracting basins of the external roots, while red corresponds to the attracting basin of the internal root $\alpha$. We use pink to emphasize the immediate basin of attraction of the internal root. Range of the phase planes $[-1.5,1.5] \times[-1.5,1.5]$. We also show in $\mathbf{c}$ and $\mathbf{d}$ the graph of each polynomial (color figure online)

Easily $T=\left(T_{1}, T_{2}\right): E_{T} \rightarrow E_{T}$ defines a smooth dynamical system given by the iterates of $T$; that is $\left\{\left(x_{m}, y_{m}\right):=T^{m}\left(x_{0}, y_{0}\right)\right\}_{m \geq 0}$ with $\left(x_{0}, y_{0}\right) \in E_{T}$ (see [5] for details). Clearly $T$ sends points of $\delta_{T}$ to infinity unless $N$ also vanishes. At those points, the definition of $T$ is uncertain in the sense that the value depends on the path we choose to approach the point. As we will see, they play a crucial role on the local and global dynamics of $T$. 

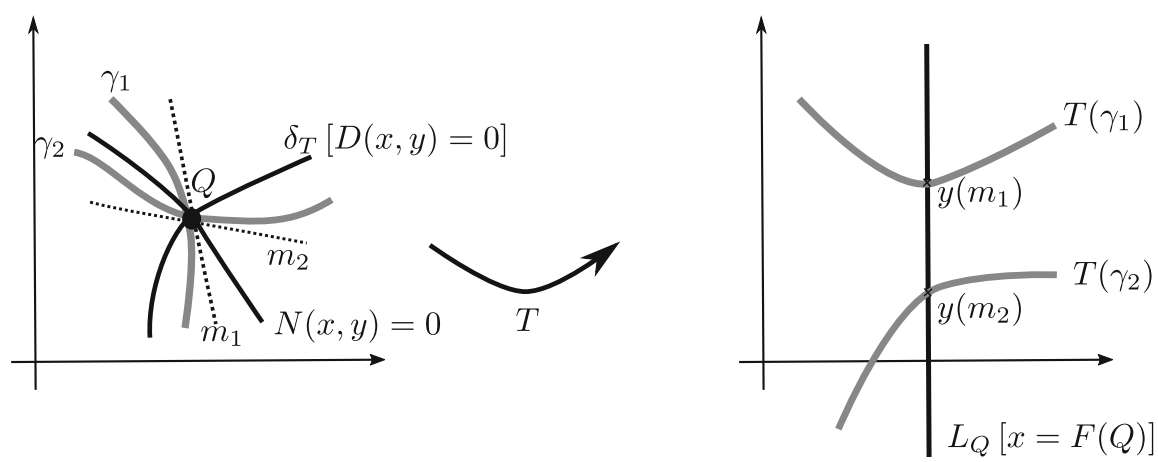

Figure 4. Dynamics of $T$ near a simple focal point $Q$

We say that a point $Q \in \delta_{T} \subset \mathbb{R}^{2}$ is a focal point (of $T$ ) if $T_{2}(Q)$ takes the form $0 / 0$ (i.e. $N(Q)=D(Q)=0$ ), and there exists a smooth simple arc $\gamma:=\gamma(t), t \in(-\varepsilon, \varepsilon)$, with $\gamma(0)=Q$, such that $\lim _{t \rightarrow 0} T_{2}(\gamma)$ exists and is finite. Moreover, the straight line given by $L_{Q}=\left\{(x, y) \in \mathbb{R}^{2} \mid x=F(Q)\right\}$ is called the prefocal line (over $Q$ ).

Let $\gamma$ passing through $Q$, not tangent to $\delta_{T}$, with slope $m$ (that is $\gamma^{\prime}(0)=$ $m)$. Then $T(\gamma)$ will be a curve passing, at $t=0$, through some finite point $(F(Q), y(m)) \in L_{Q}$. If $Q$ is simple (that is, $N_{x}(Q) D_{y}(Q)-N_{y}(Q) D_{x}(Q) \neq 0$ ) then there is a one-to-one correspondence between the slope $m$ and points in the prefocal line $L_{Q}=\left\{(x, y) \in \mathbb{R}^{2} \mid x=F(Q)\right\}$. Precisely (Fig. 4 illustrates the one-to-one correspondence),

$$
y(m)=\frac{N_{x}(Q)+m N_{y}(Q)}{D_{x}(Q)+m D_{y}(Q)} \quad \text { or } \quad m(y)=\frac{D_{x}(Q) y-N_{x}(Q)}{N_{y}(Q)-D_{y}(Q) y} .
$$

Among other dynamical aspects, simple focal points are responsible of the presence of lobes and crescents in the phase space of noninvertible maps, and in particular in the phase plane of the secant map (see Fig. 1). This kind of phenomena occurs when a basin of attraction intersects the prefocal line. Again, we refer to $[2-4]$ for other details.

Remark 1. The name focal point used here to refer the points where the map $T$ is uncertain is also known as points of indeterminacy in complex and geometric analysis.

In Fig. 5, we sketch the mechanism for the creation of lobes in the phase plane of a noninvertible map with denominator. If there exists an arc $\gamma$ crossing the prefocal line $L_{Q}$ in two different points $y\left(m_{1}\right)$ and $y\left(m_{2}\right)$, then a preimage of $T$ has a lobe issuing from the focal point $Q$. If the map has two inverses and two focal points, we can have two different lobes $T_{a}^{-1}(\gamma)$ and $T_{b}^{-1}(\gamma)$ issuing from $Q_{a}$ and $Q_{b}$. Also notice that if $\gamma$ is a lobe crossing the prefocal line $L_{Q}$ in one point $y(m)$, then an inverse $T^{-1}(\gamma)$ gives also a lobe from a focal point $Q$ but with two arcs having the same tangent $m$.

In [5], the authors used this approach to study the particular case of the secant map, that is when $T=S$, defined in (2), under the assumption that 

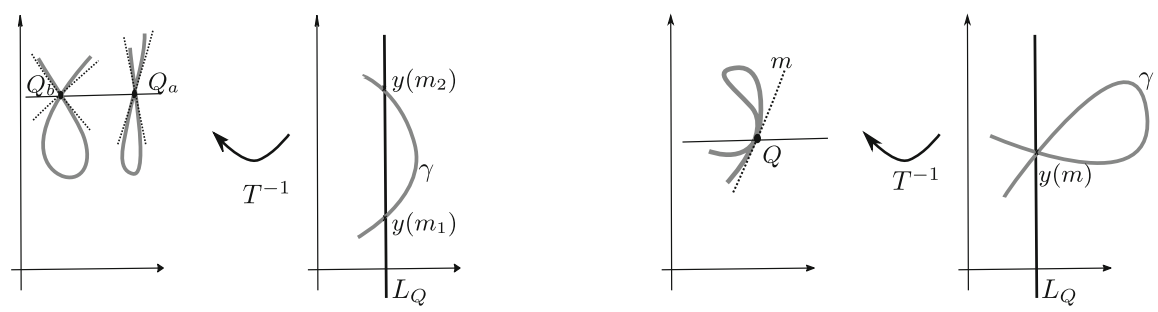

Figure 5. We sketch the mechanism responsible of the creation of lobes at focal points of a map with denominator

all real roots of $p$ are simple. In particular, it was shown that the equation

$$
p(x)-p(y)=q(x, y)(x-y),
$$

defines $q$ as a polynomial (that is, $x-y$ divides the polynomial $p(x)-p(y)$ ). Therefore, the secant map can also be written as

$$
S(x, y)=\left(y, \frac{y q(x, y)-p(y)}{q(x, y)}\right) .
$$

Moreover, for the secant map, the set $\delta_{T}$ reduces to

$$
\delta_{S}=\left\{(x, y) \in \mathbb{R}^{2} ; x \neq y \text { and } p(x)=p(y)\right\} \cup\left\{(x, x) \in \mathbb{R}^{2} ; p^{\prime}(x)=0\right\},
$$

and focal points are given by $Q_{i, j}=\left(\alpha_{i}, \alpha_{j}\right)$ with $i \neq j$ running over all possible pairs of the roots of $p$. Easily, the prefocal line of $Q_{i, j}$ is the vertical line $L_{j}=\left\{(x, y) \in \mathbb{R}^{2} ; x=\alpha_{j}\right\}$. The one-to-one correspondence at the focal point $Q_{i, j}$ described in (5) is written as

$$
y(m)=\frac{\alpha_{j} p^{\prime}\left(\alpha_{i}\right)-\alpha_{i} p^{\prime}\left(\alpha_{j}\right) m}{p^{\prime}\left(\alpha_{i}\right)-p^{\prime}\left(\alpha_{j}\right) m} \quad \text { or } \quad m(y)=\frac{p^{\prime}\left(\alpha_{i}\right)\left(\alpha_{j}-y\right)}{p^{\prime}\left(\alpha_{j}\right)\left(\alpha_{i}-y\right)} .
$$

\section{Periodic Orbits of Minimal Period 4}

It can be proved that the fixed points of the secant map applied to the polynomial $p$ are given by the points $(\alpha, \alpha)$, where $\alpha$ is a root of $p$, and that they are all attracting. It is also known (see $[1,5])$ that the secant map has no periodic orbits of period two and three in the plane although every critical point $c$ (i.e., $p^{\prime}(c)=0$ ) has associated a periodic orbit of period three given by

$$
(c, c) \stackrel{S}{\longrightarrow}(c, \infty) \stackrel{S}{\longrightarrow}(\infty, c) \stackrel{S}{\rightarrow}(c, c)
$$

after properly extending $S$ to $\infty$. Hence, it is natural to study the relevance of the period 4 orbits in the global dynamics. It is already known that those periodic orbits might be attracting. See $[1,5]$ for precise statements.

In this section, we study in detail the possible configurations of the period four orbits or 4-cycles, a key step to understand the boundary of the 
Table 1. All possible configurations of a 4-cycle and their corresponding type. Here $\star$ means incompatible configuration with a 4-cycle. See Proposition 3.1

\begin{tabular}{lllll}
\hline$\lambda>0$ & $a<b<c<d$ & $\star$ & $a<d<c<b$ & $\star$ \\
& $a<b<d<c$ & Type I & $a<c<d<b$ & Type II \\
$\lambda<0$ & $a<d<b<c$ & Type III & $a<c<b<d$ & Type IV \\
\hline
\end{tabular}

immediate basin of attraction of the fixed points of $S$. Assume that $S$ has a periodic orbit of (minimal) period 4 given by

$$
(a, b) \stackrel{S}{\rightarrow}(b, c) \stackrel{S}{\longrightarrow}(c, d) \stackrel{S}{\longrightarrow}(d, a) \stackrel{S}{\rightarrow}(a, b),
$$

where $a, b, c, d$ are real numbers. Under this notation, we are describing the dynamics of the 4-cycle (as points in $\mathbb{R}^{2}$ ), but notice that we are not determining a priory the relative position in $\mathbb{R}$ of the points $a, b, c$ and $d$ involved in the 4-cycle. In fact, the relative position among them will rise to 4 types of 4-cycles (up to symmetries; see Proposition 3.1). However, renaming points in the 4-cycle, we can assume that $a$ is the value in the cycle with minimum value, that is, we can assume without loss of generality that $a:=\min \{a, b, c, d\}$ and the dynamics of the cycle is still given by (10).

We recall that if $a, b, c, d$ are real numbers, then the cross ratio, $\lambda_{(a, b ; c, d)}$, is given by the expression

$$
\lambda:=\lambda_{(a, b ; c, d)}=\frac{(c-a)(d-b)}{(c-b)(d-a)} .
$$

Easy computations show that

$$
\lambda_{(a, d ; c, b)}=\frac{\lambda}{\lambda-1} \quad \text { and } \quad \lambda_{(d, c ; b, a)}=\lambda .
$$

The next proposition classifies completely the possible types of 4-cycles (see Fig. 6) depending on the relative position of the base points. We remark once again that the values $\{a, b, c, d\}$ are located in the real line $(\mathbb{R})$, not in the phase space $\left(\mathbb{R}^{2}\right)$.

Proposition 3.1. (Classification of 4-cycles) Assume that the secant map $S$ exhibits a 4-cycle as in (10). Then $\lambda=(-1+\sqrt{5}) / 2$ or $\lambda=-(1+\sqrt{5}) / 2$. The possible configurations (i.e, the relative position in $\mathbb{R}$ of the points a, $b, c, d$ involved in the cycle and their images by $p$ ) are listed in Table 1 and leads to four different types as described in Fig. 6. Moreover, the four types of 4-cycles are admissible.

Proof. Using the definition of the secant map and the configuration given in (10), we easily have that

$$
\begin{array}{ll}
c=b-p(b) \frac{b-a}{p(b)-p(a)}, & d=c-p(c) \frac{c-b}{p(c)-p(b)}, \\
a=d-p(d) \frac{d-c}{p(d)-p(c)}, & b=a-p(a) \frac{a-d}{p(a)-p(d)},
\end{array}
$$


$\lambda=\frac{\sqrt{5}-1}{2}>0$

$\lambda=-\frac{\sqrt{5}+1}{2}<0$
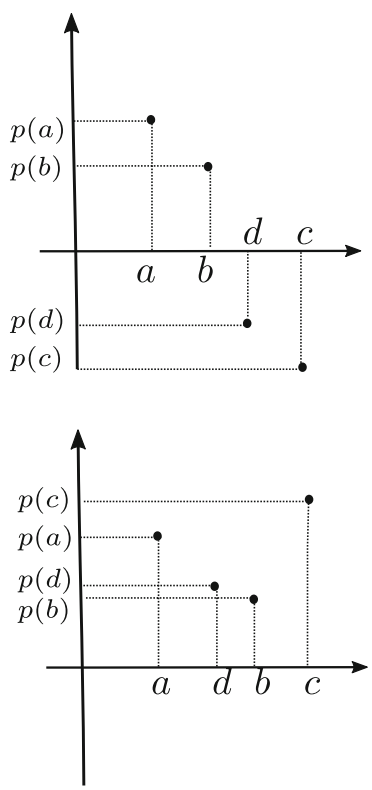
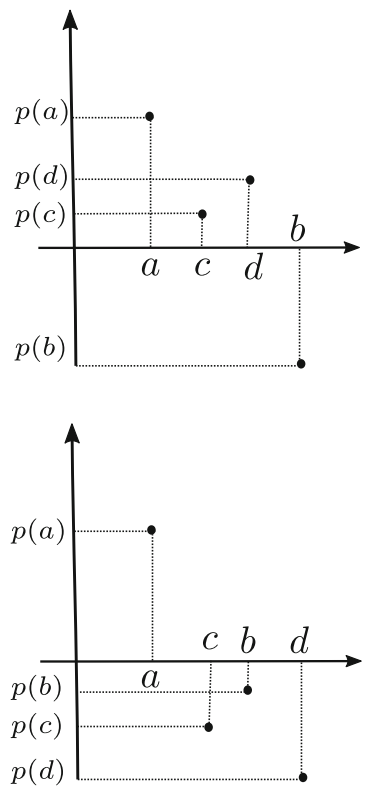

Figure 6. The four different types of 4-cycles for the secant map. We show type I in top-left and type II in top-right, corresponding to a cross ratio $\lambda>0$. We show type III in bottom-left and type IV in bottom-right, corresponding to cross ratio $\lambda<0$

which is equivalent to

$$
\frac{p(a)}{p(b)}=\frac{c-a}{c-b}, \quad \frac{p(b)}{p(c)}=\frac{d-b}{d-c}, \quad \frac{p(c)}{p(d)}=\frac{a-c}{a-d}, \quad \frac{p(d)}{p(a)}=\frac{b-d}{b-a} .
$$

Multiplying both sides of these four equations, we obtain that

$$
1=-\left[\frac{(c-a)(b-d)}{(c-d)(b-a)}\right]\left[\frac{(b-d)(a-c)}{(b-c)(a-d)}\right]=-\lambda_{(a, d ; c, b)} \lambda_{(d, c ; b, a)}=\frac{\lambda^{2}}{1-\lambda},
$$

and so $\lambda \in\{(-1+\sqrt{5}) / 2,(-1-\sqrt{5}) / 2\}$.

Now, we turn the attention to the classification of a 4-cycle of the secant map. First, let us notice the following property of the secant map. Given two points $x_{0}<y_{0}$, the secant map is given by $S\left(x_{0}, y_{0}\right)=\left(y_{0}, z_{0}\right)$, where $\left(z_{0}, 0\right)$ is the intersection between the line passing through the points $\left(x_{0}, p\left(x_{0}\right)\right)$ and $\left(y_{0}, p\left(y_{0}\right)\right)$, and the horizontal line $y=0$. Thus, if $z_{0} \in\left(x_{0}, y_{0}\right)$, then $p\left(x_{0}\right) p\left(y_{0}\right)<0$; while if $z_{0} \notin\left(x_{0}, y_{0}\right)$, then $p\left(x_{0}\right) p\left(y_{0}\right)>0$.

We need to consider 6 cases depending on the relative position of the points $a, b, c, d$ on the real line since we have assumed that $a<\min \{b, c, d\}$. It follows from the definition of the cross ratio $\lambda(a, b ; c, d)(11)$ that $\lambda$ is positive if and only if one and only one of $c$ and $d$ lays between $a$ and $b$. So, there are four cases where $\lambda>0$ and two cases where $\lambda<0$.

Case 1. $a<b<c<d(\lambda>0)$. We have $S(a, b)=(b, c)$ and $c \notin(a, b)$. So $p(a) p(b)>0$. Since $S(b, c)=(c, d)$ and $b<c<d$ we get $p(c) p(b)>0$. Also, 
since $S(c, d)=(d, a)$ and $a \notin(c, d)$, we get $p(a) p(b) p(c) p(d)>0$. Finally, since $S(d, a)=(a, b)$ and $b \in(a, d)$ we have $p(a) p(d)<0$, a contradiction. Thus, there are no 4-periodic orbits with this configuration.

Case 2. $a<b<d<c(\lambda>0)$. We have $S(a, b)=(b, c)$ with $c>b$. So $p(a) p(b)>0$ (we assume $p(a)>p(b)>0$, the case $p(a)<p(b)<0$ follows similarly). Since $S(b, c)=(c, d)$ and $d \in(b, c)$ then $p(c)<0$ (we have assumed $p(b)>0)$. Also we have $S(c, d)=(d, a)$ and since $a<d<c$ then $p(c)<p(d)<0$. Finally, $S(d, a)=(a, b)$ which is compatible with the fact that $p(a) p(d)<0$. This 4 -cycle corresponds to type I. See Fig. 6 (first row left).

Case 3. $a<c<d<b(\lambda>0)$. We have $S(a, b)=(b, c)$ with $c \in(a, b)$. So $p(a) p(b)<0$ (moreover, assuming that $p(a)>0$, we have that $p(b)<0$; the case $p(a)<0$ follows similarly). Since $S(b, c)=(c, d)$ and $d \in(c, b)$, we have $p(c)>0$. Since $S(c, d)=(d, a), p(c)>0$ and $a<c<d$, we have $p(d)>p(c)>0$. Finally, since $S(d, a)=(a, b)$ with $a<d<b$, we get $p(a)>p(d)>p(c)>0$ and $p(b)<0$, a compatible configuration which corresponds to type III. See Fig. 6 (first row right).

Cases 4. $a<d<c<b(\lambda>0)$. This case leads to an incompatible configuration and we leave the details to the reader.

Case 5. $a<d<b<c(\lambda<0)$. We have $S(a, b)=(b, c)$ with $a<b<c$. So $p(a) p(b)>0$ (moreover, assuming that $p(b)>0$, we have that $p(a)>p(b)$; the case $p(b)<0$ follows similarly). Since $S(b, c)=(c, d)$ and $d \notin(b, c)$ we conclude that $p(c)>p(b)>0$. Since $S(c, d)=(d, a)$ and $a<d<c$ we have $0<p(d)<p(c)$. Hence $p(a), p(b), p(c)$ and $p(d)$ are all positive. Finally, since $S(d, a)=(a, b)$ with $a<b<d$, we conclude that this configuration is possible and corresponds to type $I I$ (the case $p(b)<0$ is symmetric with $p(a), p(b), p(c)$ and $p(d)$ all negative). See Fig. 6 (second row left).

Case 6. $a<c<b<d(\lambda<0)$. We have $S(a, b)=(b, c)$ with $c \in(b, a)$. So $p(a) p(b)<0$ (moreover, assuming that $p(a)>0$, we have that $p(b)<0$; the case $p(a)<0$ follows similarly). Since $S(b, c)=(c, d)$ and $d \notin(c, b)$, we have $p(c)<p(b)<0$. Since $S(c, d)=(d, a), p(c)<0$ and $a<c<d$, we have $p(d)<p(c)<0$. Finally, since $S(d, a)=(a, b)$ with $a<b<d$, we get $p(d)<p(c)<p(b)<0$ and $p(a)>0$, a compatible configuration which corresponds to type III. See Fig. 6 (second row right).

We finally show that the four different types of 4-cycles are admissible. In fact we show how to numerically built a concrete polynomial having a 4-cycle of Type I and we leave the details of the other cases to the reader since the strategy is quite similar.

We choose the configuration: $a<b<d<c$ which corresponds to $\lambda>0$. We fix $a=1, b=2$ and $c=3$. Since we know that $\lambda=(\sqrt{5}-1) / 2$ we get $d \approx 2.447213595$. Now we need to determine the value of $p(a), p(b), p(c)$ and $p(d)$ so that (10) is satisfied. From (13) we can easily compute $p(a), p(b), p(c)$ and $p(d)$. Indeed it is an homogeneous linear system of equations with one degree of freedom. So fixing $p(d)=-1$ we obtain $p(a) \approx 2.23606798, p(b) \approx$ 1.118033989 and $p(c) \approx-1.381966011$. Finally, we use Newton interpolation to get 


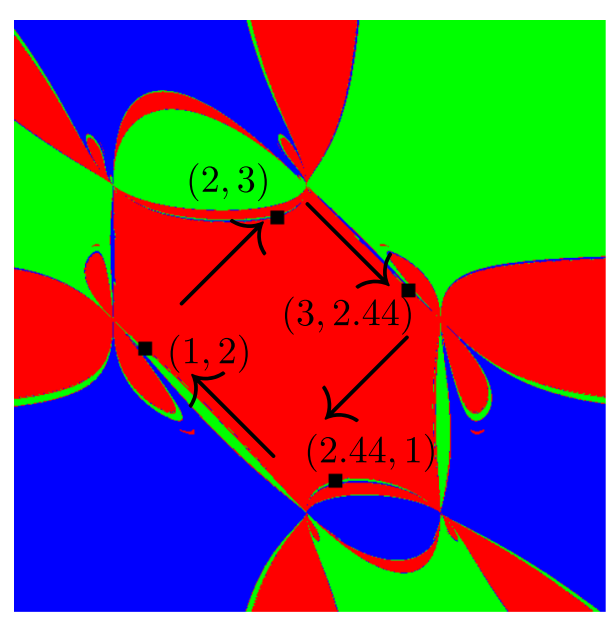

Figure 7. Phase plane of the secant map applied to the polynomial $p^{I}$. We denote each point in the 4 -cycle $(1,2) \stackrel{S}{\longrightarrow}$ $(2,3) \stackrel{S}{\longrightarrow}(3,2.447213595) \stackrel{S}{\longrightarrow}(2.447213595,1) \stackrel{S}{\longrightarrow}(1,2)$ with an small black square

$$
\begin{aligned}
p^{I}(x)= & 2.23606798-1.11803390(x-1)-0.6909830(x-1)(x-2) \\
& +3.27254249(x-1)(x-2)(x-3) .
\end{aligned}
$$

According to the arguments above the secant map $S_{P^{I}}$ has a 4-cycle of Type I (see Fig. 7). Similarly $S_{P^{I I}}, S_{P^{I I I}}$ and $S_{P^{I V}}$ have 4-cycle of Type II, III and $\mathrm{IV}$, respectively, where

$$
\begin{gathered}
p^{I I}(x)=2.818-5.236(x-1)+4.3316(x-2)(x-1)-16.106(x-2)(x-1)(x-3) \\
p^{I I I}(x)=2.236-1.118(x-1)+1.809(x-2)(x-1)-0.4774(x-2)(x-1)(x-3) \\
p^{I V}(x)=1.618-2.118(x-1)+0.809(x-2)(x-1)-1.7135(x-2)(x-1)(x-3) .
\end{gathered}
$$

In Fig. 7, we show the phase plane of the secant map applied to the polynomial $p^{I}$. This polynomial exhibits three roots. We also show the 4cycle

$$
(1,2) \stackrel{S}{\longrightarrow}(2,3) \stackrel{S}{\longrightarrow}(3,2.447213595) \stackrel{S}{\longrightarrow}(2.447213595,1) \stackrel{S}{\longrightarrow}(1,2) .
$$

Every point in the 4-cycle of Type I is shown in the picture with a small black square and we will see in the next sections the crucial role of this 4-cycle with the basin of attraction of the internal root of $p^{I}$.

We believe that 4-cycles are crucial to understand the global phase portrait and the structure of the boundary of the immediate basins of attraction (internal and external). We conjecture that different types of 4-cycles control different types of roots of $p$. More precisely, the boundary of an internal root contains a period 4 orbit of type I (see Theorems A and B); the boundary 
of an external root of a polynomial of even degree contains a period 4 orbit of type II or IV; and the boundary of an internal multiple root of even multiplicity contains a period 4 orbit of type III.

\section{Proof of Theorem A}

First, we prove the topological description of the boundary of the immediate basin of attraction of an internal root, that is Theorem A(a). At the end of the section, we prove Theorem $\mathrm{A}(\mathrm{b})$.

Hereafter we fix the following notation. We assume, without lost of generality, that $\alpha_{0}<\alpha_{1}<\alpha_{2}$ are three consecutive real simple roots of $p$ and $p^{\prime}\left(\alpha_{0}\right)>0, p^{\prime}\left(\alpha_{1}\right)<0$ and $p^{\prime}\left(\alpha_{2}\right)>0$. So $p(x)>0$ for all $x \in\left(\alpha_{0}, \alpha_{1}\right)$ and $p(x)<0$ for all $x \in\left(\alpha_{1}, \alpha_{2}\right)$. Moreover, $p$ should have at least one critical point in each open interval $\left(\alpha_{0}, \alpha_{1}\right)$ and $\left(\alpha_{1}, \alpha_{2}\right)$. We denote by $c_{1}$ the largest critical point of $p$ in $\left(\alpha_{0}, \alpha_{1}\right)$ and by $c_{2}$ the smallest critical point of $p$ in $\left(\alpha_{1}, \alpha_{2}\right)$ (equivalently the open interval $\left(c_{1}, c_{2}\right)$ is free of critical points). Of course $\alpha_{1}$ is the target internal root of Theorem A.

Following the notation of Sect. 2 (see also [5]), one can show that the focal points of $S$ are given by $Q_{i, j}=\left(\alpha_{i}, \alpha_{j}\right), i \neq j \in\{0,1,2\}$, and that each $Q_{i, j}$ has the vertical line $L_{j}=\left\{(x, y) \in \mathbb{R}^{2} \mid x=\alpha_{j}\right\}$ as its prefocal line. Moreover, we also known that $\mathcal{A}^{*}\left(\alpha_{1}\right)$ is bounded. Next lemma makes this condition more precise.

Lemma 4.1. Let $\alpha_{0}<\alpha_{1}<\alpha_{2}$ be three real simple consecutive roots of $p$. Then $\mathcal{A}^{*}\left(\alpha_{1}\right) \subset R$ where $R:=\left\{(x, y) \in \mathbb{R}^{2} \mid \alpha_{0}<x<\alpha_{2}, \alpha_{0}<y<\alpha_{2}\right\}$.

Proof. From (2), it is easy to see that given any root $\alpha \in \mathbb{R}$ of $p$ we have $S(x, \alpha)=(\alpha, \alpha)$ and $S(\alpha, y)=(y, \alpha)$, as long as $x$ and $y$ are not roots of $p$. This implies that

$$
\left(\partial R \backslash \bigcup_{i \neq j \in\{0,1,2\}} Q_{i, j}\right) \subset\left(A^{\star}\left(\alpha_{0}\right) \cup A^{\star}\left(\alpha_{2}\right)\right) .
$$

Since the focal points $Q_{i, j}$ belong to $\delta_{S}$ where $S$ is not even defined the lemma follows. See Fig. 8 .

We define the external boundary of $\mathcal{A}^{*}\left(\alpha_{1}\right)$ as follows. Consider $U$ the open set $\mathbb{C} \backslash \overline{\mathcal{A}^{*}\left(\alpha_{1}\right)}$ and let $V$ be the unique unbounded connected component of $U$. Then, the external boundary of $\mathcal{A}^{*}\left(\alpha_{1}\right)$ is $\partial V$. Notice that $V$ is unique since $\mathcal{A}^{*}\left(\alpha_{1}\right)$ is bounded (see Lemma 4.1$)$. We will assume that the external boundary of $\mathcal{A}^{*}\left(\alpha_{1}\right)$ is piecewise smooth; i.e., a union of smooth arcs (i.e., diffeomorphic to $(0,1))$ joining the focal points.

Proposition 4.2. Let $p$ be a polynomial and let $\alpha_{0}<\alpha_{1}<\alpha_{2}$ be three consecutive simple roots of $p$. Assume the external boundary of $\mathcal{A}^{*}\left(\alpha_{1}\right)$ is piecewise smooth. Then, $\partial \mathcal{A}^{*}\left(\alpha_{1}\right)$ contains a smooth hexagon-like polygon with $C^{1}$-lobes where the vertices are the focal points $Q_{1,0}, Q_{2,0}, Q_{0,1}, Q_{2,1}, Q_{0,2}$ and $Q_{1,2}$, and lobes are issuing only from to $Q_{1,0}, Q_{2,0}, Q_{0,2}$ and $Q_{1,2}$. 


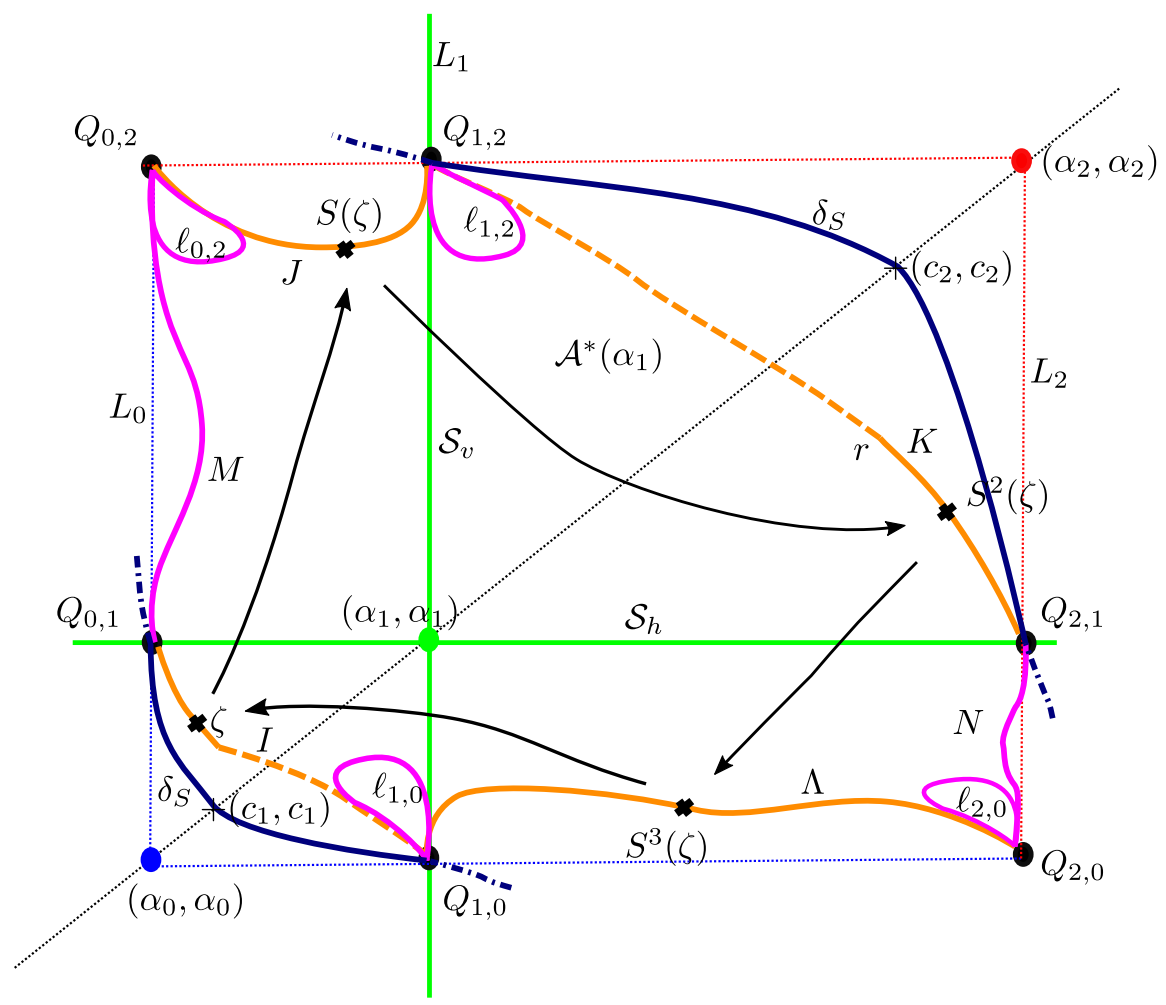

Figure 8. Sketch of external boundary of the immediate basin of attraction of an internal root $\alpha_{1}$. In the picture, we can see the six focal points $Q_{i, j}$, where $i, j$ are two different numbers in $\{0,1,2\}$ and the 4 -cycle $\zeta \rightarrow S(\zeta) \rightarrow S^{2}(\zeta) \rightarrow$ $S^{3}(\zeta) \rightarrow \zeta$

Proof. We will assume, without lost of generality, that $p^{\prime}\left(\alpha_{0}\right)>0$ (and so $p^{\prime}\left(\alpha_{1}\right)<0$ and $\left.p^{\prime}\left(\alpha_{2}\right)>0\right)$.

Focal points do not belong to $\mathcal{A}^{*}\left(\alpha_{1}\right)$, while from Lemma 4.1 it follows that the segments $S_{v}:=\left\{\left(\alpha_{1}, y\right) ; \alpha_{0}<y<\alpha_{2}\right\}$ and $S_{h}:=\left\{\left(x, \alpha_{1}\right) ; \alpha_{0}<\right.$ $\left.x<\alpha_{2}\right\}$ do. In particular, we have that $\left\{Q_{0,1}, Q_{2,1}, Q_{1,0}, Q_{1,2}\right\} \in \partial \mathcal{A}^{*}\left(\alpha_{1}\right)$. Since $\mathcal{A}^{*}\left(\alpha_{1}\right) \subset R$ (see Lemma 4.1) and the external boundary of $\mathcal{A}^{*}\left(\alpha_{1}\right)$ is piecewise smooth there should be an arc $I \subset \partial \mathcal{A}^{*}\left(\alpha_{1}\right)$ joining $Q_{1,0}$ and $Q_{0,1}$ and an arc $K \subset \partial \mathcal{A}^{*}\left(\alpha_{1}\right)$ joining $Q_{1,2}$ and $Q_{2,1}$ belonging to the external boundary.

We claim that $S(I)$ is an arc $J \subset \partial \mathcal{A}^{*}\left(\alpha_{1}\right)$ connecting the focal points $Q_{0,2}$ and $Q_{1,2}$. To see the claim we notice that when $I$ approaches $Q_{0,1}$ (with negative slope by construction; see Fig. 8) its image should be an arc landing at $L_{1} \cap \partial \mathcal{A}^{*}\left(\alpha_{1}\right)$. Since $\mathcal{A}^{*}\left(\alpha_{1}\right) \subset R$ and $L_{1} \cap R \subset \mathcal{A}^{*}\left(\alpha_{1}\right)$ we conclude that the landing point should be either $Q_{1,2}$ or $Q_{1,0}$. Using the one-to-one correspondence defined in (9), it is clear that the landing point cannot be $Q_{1,0}$ because this corresponds to $m=\infty$. Similarly we can show that when $I$ 
approaches $Q_{1,0}$ (again with negative slope by construction) its image should be an arc landing at $Q_{0,2}$. Moreover $J \subset R$ since, by Lemma 4.1, we have that $\partial R \cap \mathcal{A}^{*}\left(\alpha_{1}\right)=\emptyset$.

Arguing similarly on $K$ instead of $I$, we see that $\Lambda:=S(K)$ is a smooth arc joining $Q_{1,0}$ and $Q_{2,0}$ entirely contained in $R$ as it is illustrated in Fig. 8.

Finally, since $\mathcal{A}^{*}\left(\alpha_{1}\right) \subset R$ and the assumption on the smoothness of the external boundary of $\mathcal{A}^{*}\left(\alpha_{1}\right)$ there should be two arcs, one denoted by $N$ joining $Q_{2,0}$ and $Q_{2,1}$ and another denoted by $M$ joining $Q_{0,1}$ and $Q_{0,2}$, with $\{N, M\} \subset \partial \mathcal{A}^{*}\left(\alpha_{1}\right)$ belonging to $R$.

Since $N$ is an arc issuing from two focal points $Q_{2,0}$ and $Q_{2,1}$, its image $S(N)$ must be an arc issuing from the prefocal line of the two focal points, which are $L_{0}$ and $L_{1}$. Moreover, since the two focal points $Q_{2,0}$ and $Q_{2,1}$ belong to the line $L_{2}$ which is mapped into $y=\alpha_{2}$ we have that necessarily the arc $S(N)$ connects the focal points $Q_{0,2}$ and $Q_{1,2}$ so that it must be $J=S(N)=S(I)$. Reasoning in a similar way, we can state that the image of the $\operatorname{arc} M$, must be $\Lambda=S(M)=S(K)$.

Up to this point we have constructed an hexagon-like polygon without lobes formed by six smooth $\operatorname{arcs} I, J, K, \Lambda, M$ and $N$ with vertices at the focal points $Q_{1,0}, Q_{0,1}, Q_{0,2}, Q_{1,2}, Q_{2,1}$ and $Q_{2,0}$ contained in $\partial \mathcal{A}^{*}\left(\alpha_{1}\right)$. Of course the hexagon (without the vertices) is forward invariant and $I \rightarrow J, N \rightarrow J$, $K \rightarrow \Lambda$ and $M \rightarrow \Lambda$. Moreover, observe that each curve approaching $Q_{0,1}$ inside the internal sector defined by the $\operatorname{arcs} I$ and $M$ (for instance $S_{h}$ ) will be sent to a curve through a point in $S_{v}$ so contained in $A^{\star}\left(\alpha_{1}\right)$. Hence no curve in this sector might be in $\partial A^{\star}\left(\alpha_{1}\right)$. Similarly for the focal point $Q_{2,1}$ in the internal sector defined by $K$ and $N$.

The arc $J$ is issuing from $Q_{0,2}$ and $Q_{1,2}$ and its image must be also on the boundary, issuing from points of the prefocal $L_{2}$. However, its image cannot be the $\operatorname{arc} N$ since this would lead a two-cyclic set implying the existence of a 2-cycle which is impossible. Thus, the arc issuing from $Q_{0,2}$ and the arc issuing from $Q_{1,2}$ are both mapped into an arc issuing from $Q_{2,1}$, which means that the image of $J$ is folded on a portion of the $\operatorname{arc} K$, and a folding point $r_{K}$ must exist on $K$. Similarly for the other arc $\Lambda$, its image is folded on an arc of $I$ issuing from $Q_{0,1}$.

Finally, taking preimages of the $\operatorname{arcs} N$ and $M$, we obtain countable many lobes attached at the four focal points $Q_{1,0}, Q_{2,0}, Q_{0,2}$ and $Q_{1,2}$. See Fig. 8. We briefly show the inductive construction of this sequence of lobes. The arc $M$ connects two points in the prefocal line $L_{0}$; hence, the preimage of $M$ should be given by a lobe issuing from a related focal point (see the qualitative picture in Fig. 5a). In our case, we have two focal points both having the prefocal line $L_{0}$, which are $Q_{1,0}$ and $Q_{2,0}$; thus, we have two preimages of $M$ giving two lobes issuing from these two focal points. We denote by $\ell_{1,0}$ and $\ell_{2,0}$ the lobes attached to the focal points $Q_{1,0}$ and $Q_{2,0}$, respectively. Similarly, we can construct the other two lobes (as preimages of N) $\ell_{0,2}$ and $\ell_{1,2}$ attached to $Q_{0,2}$ and $Q_{1,2}$.

Now, we can take the preimages of the lobe $\ell_{0,2}$, since it is issuing from the prefocal line $L_{0}$ its preimage should be given by a lobe issuing from a related focal point (see the qualitative picture in Fig. 5b). In our case, we 


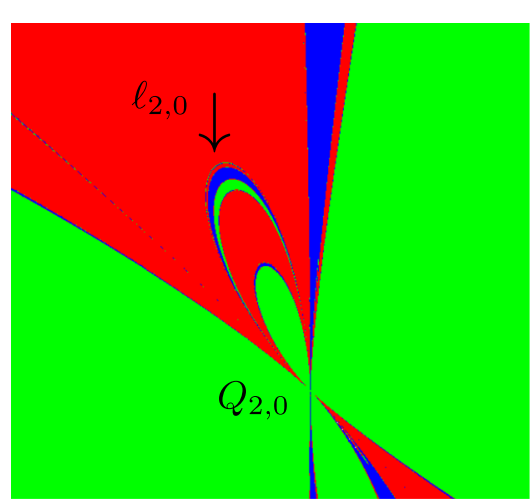

(a) The lobe $\ell_{02}$ attached to the focal point $Q_{2,0}$.

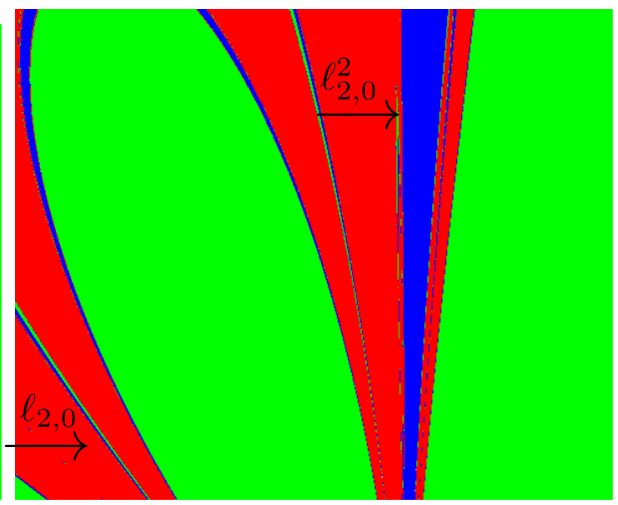

(b) The lobe $\ell_{2,0}^{2}$ attached to the focal point $Q_{2,0}$.

Figure 9. Enlargement of a portion of the phase plane of the secant map applied to $T_{3}(x)=4 x^{3}-3 x$ near the focal point $Q_{2,0}$

have two focal points both having the prefocal line $L_{0}$, which are $Q_{1,0}$ and $Q_{2,0}$; thus, we have two preimages of the lobe $\ell_{0,2}$ giving two lobes issuing from these two focal points, say $\ell_{1,0}^{2}$ and $\ell_{2,0}^{2}$.

In the same way, we can prove the existence of two lobes $\ell_{0,2}^{2}$ and $\ell_{1,2}^{2}$ issuing from the focal points $Q_{0,2}$ and $Q_{1,2}$ as preimages of the lobe $\ell_{2,0}$. Inductively, each lobe $\ell_{2,0}^{n}$ issuing from the focal point $Q_{2,0}$ has preimages in two lobes $\ell_{0,2}^{n+1}$ and $\ell_{1,2}^{n+1}$ issuing from the focal points $Q_{0,2}$ and $Q_{1,2}$, and $\ell_{0,2}^{n}$ issuing from the focal point $Q_{0,2}$ has preimages in two lobes $\ell_{1,0}^{n+1}$ and $\ell_{2,0}^{n+1}$ issuing from the focal points $Q_{1,0}$ and $Q_{2,0}$. Notice that the lobes issuing from $Q_{1,0}$ and $Q_{1,2}$ have not preimages internal to the immediate basin, because such preimages are issuing from the focal points $Q_{0,1}$ and $Q_{2,1}$ and we have shown that lobes cannot exist inside the external boundary detected above, so that the related preimages must be outside the external boundary.

In Fig. 9, we show the phase plane of the secant map applied to the Chebyshev polynomial $T_{3}$ near the focal point $Q_{2,0}$. In this picture we can see the lobe $\ell_{20}$ which is a preimage of $M$ (Fig. 9, left) and the lobe $\ell_{2,0}^{2}$ attached to the focal point $Q_{2,0}$ with slope equal to $\infty$ (Fig. 9, right).

Corollary 4.3. Let $p$ be a polynomial and let $\alpha_{0}<\alpha_{1}<\alpha_{2}$ be three consecutive real simple roots of $p$. Then, there exists a 4 -cycle $\mathcal{C} \in \partial \mathcal{A}^{*}\left(\alpha_{1}\right)$ of type I.

Proof. According to the arguments used in the proof of Proposition 4.2 we know that for the arc-edge $I$ of the hexagon-like polygon we have $S^{4}: I \rightarrow$ $I_{1} \subset I$, where $I_{1}$ is an arc issuing from the focal point $Q_{0,1}$. Hence, there should be a fixed point $\zeta \in I_{1}$. Of course $\mathcal{C}=\left\{\zeta, S(\zeta), S^{2}(\zeta), S^{3}(\zeta)\right\}$ is a 4-cycle of $S$ since each point belongs to a different edge of the hexagon-like border and, from Proposition 3.1, it is of type $I$. Moreover, we know that 


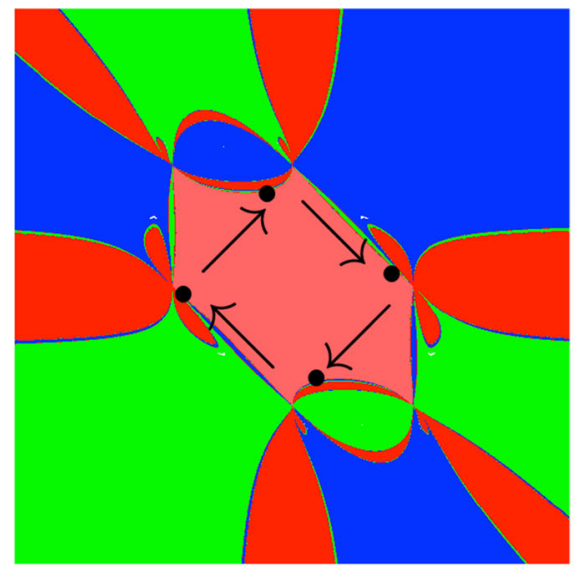

(a) $T_{3}(x)=4 x^{3}-3 x$.

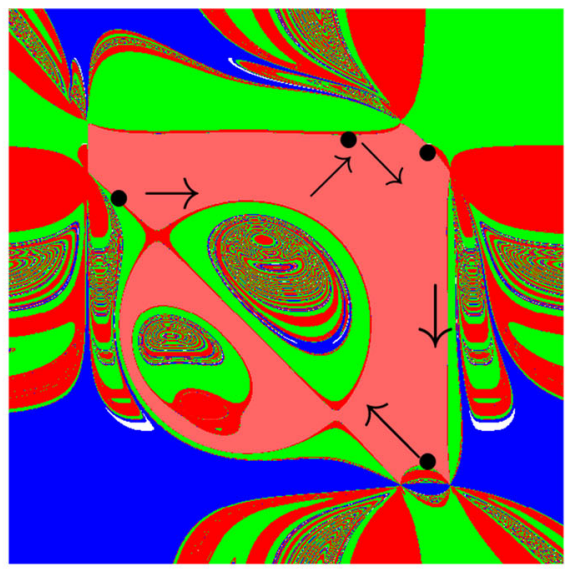

(b) $p(x)=\frac{x 5}{5}-\frac{x^{3}}{3}-0.05 x+0.15$.

Figure 10. Phase plane of the secant map applied to the Chebyshev polynomial $T_{3}$ (left) and to the polynomial $p$ (right). We show the immediate basin of attraction of the internal root in pink. We also mark the 4-cycle contained in the boundary of the immediate basin proved in Theorem A. Range of the pictures $[-2,2] \times[-2,2]$

on the transverse direction to $I$, the point $\zeta$ should be a repeller (for $S^{4}$ ) since the points near $\zeta$ outside $I$ move away from $I$, in particular the ones converging to $\left(\alpha_{1}, \alpha_{1}\right)$. Hence, $\zeta$ is a transversely repelling point for $S^{4}$.

Remark 2. We conjecture that the hypothesis on the smoothness of the external boundary of $\partial \mathcal{A}^{*}\left(\alpha_{1}\right)$ is not needed.

Remark 3. Corollary 4.3 does not claim that the period 4-cycle is a saddle point of $S^{4}$. However, we conjecture it is so with one side of its unstable 1-dimensional manifold entering on $\mathcal{A}^{*}\left(\alpha_{1}\right)$ and the stable manifold lying on $\partial \mathcal{A}^{*}\left(\alpha_{1}\right)$. As an example for this we consider the polynomial $p^{I}$ given in (14) and its 4 -cycle $\zeta=(1,2) \mapsto(2,3) \mapsto(3,2.44) \mapsto(2.44,1)$. Some computations show that

$$
D S^{4}(\zeta) \approx\left(\begin{array}{ll}
207.26 & 236.15 \\
242.42 & 276.37
\end{array}\right)
$$

with eigenvalues $\lambda_{1} \approx 483.55$ and $\lambda_{2} \approx 0.05$. So clearly $\zeta$ is a saddle point. Moreover, the corresponding eigenvectors $v_{1} \approx(-0.65,-0.76)$ and $v_{2} \approx$ $(-0.75,0.66)$ show that the unstable and stable manifolds (locally) coincide with the mentioned directions. See Fig. 7 .

Proof of Theorem A. Statement (a) follows from Proposition 4.2 while statement (b) follows from Corollary 4.3.

We notice that Theorem A applies independent of the connectedness of the immediate basin of attraction of the internal root. We present two 
examples to focus on this fact. We consider the phase space of the secant map applied to the Chebyshev polynomial $T_{3}(x)=4 x^{3}-3 x$, see Fig. 10 (left), in contrast with the phase space of the secant map applied to the polynomial $p(x)=\frac{x 5}{5}-\frac{x^{3}}{3}-0.05 x+0.15$, see Fig. 10b. In both cases, the two polynomials exhibit three simple root, and thus in both cases, there exist a unique internal root. In pink we show the immediate basin of attraction of the internal root. In the case of $T_{3}$ the immediate basin of attraction is simply connected while in the case of $p$ the immediate basin is multiply connected. Moreover, we numerically compute the 4-cycle contained in the boundary of the immediate basin of attraction as Theorem A states. Every point in the 4-cycle is depicted in the phase plane with a small black circle. Finally, we mention that Theorem A only deals with the external boundary of the immediate basin of attraction of the internal root. In the next section, we precisely focus on sufficient conditions which ensure that the immediate basin of attraction of an internal root is simply connected.

\section{Proof of Theorem B}

As in the previous section we assume, without lost of generality, that $\alpha_{0}<$ $\alpha_{1}<\alpha_{2}$ are three consecutive real simple roots of $p$ and $p^{\prime}\left(\alpha_{0}\right)>0, p^{\prime}\left(\alpha_{1}\right)<0$ and $p^{\prime}\left(\alpha_{2}\right)>0$. We denote by $R, H_{y_{0}}$ and $V_{y_{0}}$ the following open sets

$$
\begin{aligned}
& R=\left\{(x, y) \in \mathbb{R}^{2} ; \alpha_{0}<x<\alpha_{2}, \alpha_{0}<y<\alpha_{2}\right\}, \\
& H_{y_{0}}=\left\{\left(x, y_{0}\right) \in \mathbb{R}^{2} ; \alpha_{0}<x<\alpha_{2}\right\}, \\
& V_{y_{0}}=\left\{\left(y_{0}, y\right) \in \mathbb{R}^{2} ; \alpha_{0}<y<\alpha_{2}\right\} .
\end{aligned}
$$

Moreover, we introduce the auxiliary map

$$
\varphi_{y}(x)=y-\frac{p(y)}{q(x, y)},
$$

which coincides with the second component of the secant map; i.e., $S(x, y)=$ $\left(y, \varphi_{y}(x)\right)$ where remember that the polynomial $q$ was defined in (6).

We now investigate the connectedness of the basin of attraction of an internal root $\alpha_{1}$. In the next lemma, we count the number of inverses of the secant map for a given point $(x, y) \in R$. In particular this lemma will apply to points in $\mathcal{A}^{\star}\left(\alpha_{1}\right)$ (see Lemma 4.1).

Lemma 5.1. Let $p$ be a polynomial and let $\alpha_{0}<\alpha_{1}<\alpha_{2}$ be three consecutive simple real roots of $p$. Assume further that $p$ has only one inflection point in the interval $\left(\alpha_{0}, \alpha_{2}\right)$. Then for any point $(x, y) \neq\left(\alpha_{1}, \alpha_{1}\right)$ in $R$, we have that $\#\left\{S^{-1}(x, y)\right\} \leq 2$ where $S^{-1}$ means preimages of $(x, y)$ in $R$.

Proof. We reason by contradiction. We assume that there exists $\left(x_{1}, y_{1}\right) \in R$ with three different preimages in $R$, say $\left(w_{0}, x_{1}\right),\left(w_{1}, x_{1}\right)$ and $\left(w_{2}, x_{1}\right)$ so that $S\left(w_{i}, x_{1}\right)=\left(x_{1}, y_{1}\right)$ with $i=0,1,2$. Renaming these points if necessary we can assume that $w_{0}<w_{1}<w_{2}$. Let $r$ be the line passing through $\left(x_{1}, p\left(x_{1}\right)\right)$ and $\left(y_{1}, 0\right)$. By construction the points $\left(w_{i}, p\left(w_{i}\right)\right), i=0,1,2$ belong to $r$. Thus, the line $r$ contains the points $\left(x_{1}, p\left(x_{1}\right)\right)$ and $\left(w_{i}, p\left(w_{i}\right)\right), i=0,1,2$ and this implies the existence of at least two inflection points of $p$ in the interval 
defined by $\beta_{0}:=\min \left\{x_{1}, w_{0}\right\}$ and $\beta_{2}:=\max \left\{x_{1}, w_{2}\right\}$ with $\left[\beta_{0}, \beta_{2}\right] \subset\left(\alpha_{0}, \alpha_{2}\right)$, a contradiction with the assumptions.

Lemma 5.2. Let $p$ be a polynomial and let $\alpha_{0}<\alpha_{1}<\alpha_{2}$ be three consecutive simple real roots of $p$. Assume further that $p$ has only one inflection point in $\left(\alpha_{0}, \alpha_{2}\right)$ and let $y_{0} \in\left(\alpha_{0}, \alpha_{2}\right)$. Then, the set $J_{y_{0}}:=S\left(H_{y_{0}}\right) \cap \bar{R}$ is a closed vertical segment belonging to $\overline{V_{y_{0}}}$ and, if $y_{0} \neq \alpha_{1}$, any point of $J_{y_{0}}$ has two preimages in $H_{y_{0}}$, counting multiplicity. Moreover,

(a) if $y_{0}<\alpha_{1}$ then $J_{y_{0}}=\left[\varphi\left(x_{0}^{*}\left(y_{0}\right)\right), \alpha_{2}\right]$,

(b) if $y_{0}>\alpha_{1}$ then $J_{y_{0}}=\left[\alpha_{0}, \varphi\left(x_{0}^{*}\left(y_{0}\right)\right)\right]$,

(c) if $y_{0}=\alpha_{1}$ then $J_{y_{0}}=\left[\alpha_{1}, \alpha_{1}\right]$ (degenerate closed interval),

where $x_{0}^{*}\left(y_{0}\right)$ is the unique point in $H_{y_{0}}$ such that $\frac{\partial q}{\partial x}\left(x_{0}^{*}\left(y_{0}\right), y_{0}\right)=0$.

Proof. First remember that $\varphi_{y}(x)$ is defined in (15) as the second component of the secant map. Therefore, we already know that if $y_{0}=\alpha_{1}$ then $\varphi_{y_{0}}(x) \equiv$ $\alpha_{1}$ and (c) follows. In what follows, we fix a concrete value of $y_{0} \in\left(\alpha_{0}, \alpha_{2}\right)$ with $y_{0} \neq \alpha_{1}$. On the one hand from the expression of the secant map we have that $S\left(H_{y_{0}}\right) \cap \bar{R}$ is a closed vertical segment $J_{y_{0}}:=[a, b] \subset \overline{V_{y_{0}}}$. On the other hand it is a direct computation to see that

$$
\varphi^{\prime}(x)=\frac{p\left(y_{0}\right)}{q^{2}\left(x, y_{0}\right)} \frac{\partial q}{\partial x}\left(x, y_{0}\right) \quad \text { and } \quad \frac{\partial q}{\partial x}\left(x, y_{0}\right)=\frac{p^{\prime}(x)-q\left(x, y_{0}\right)}{x-y_{0}} .
$$

Observe from $(6)$ that $q(x, y)$ is a polynomial and simple computations show that when $x=y_{0}$ the second formula becomes $\partial q / \partial x\left(y_{0}, y_{0}\right)=p^{\prime \prime}\left(y_{0}\right) / 2$. Hence, $\varphi^{\prime}(x)$ vanishes if and only if $p^{\prime}(x)-q\left(x, y_{0}\right)=0$ for $x \neq y_{0}$, or $p^{\prime \prime}\left(y_{0}\right)=0$ if $x=y_{0}$.

As already said, the focal points $Q_{i, j} \in \delta_{S}$, the set of non definition of map $S$ (now we focus on $i \neq j \in\{0,1,2\}$ ). Moreover, it is easy to argue that the points $\left(c_{k}, c_{k}\right), k=1,2$ also belong to $\delta_{S} \cap R$. It follows that an arc of $\delta_{S}$ must exist in $R$ connecting the points $Q_{0,2},\left(c_{1}, c_{1}\right)$ and $Q_{1,0}$ (as qualitatively shown in Fig. 8), so that for $\alpha_{0}<y_{0}<\alpha_{1}$ the graph of $\varphi(x)$ has a vertical asymptote for $x \in\left(\alpha_{0}, \alpha_{1}\right)$. Similarly, an arc of $\delta_{S}$ must exist in $R$ connecting the points $Q_{1,2},\left(c_{2}, c_{2}\right)$ and $Q_{2,1}$ (as qualitatively shown in Fig. 8), so that for $\alpha_{1}<y_{0}<\alpha_{2}$ the graph of $\varphi(x)$ has a vertical asymptote for $x \in\left(\alpha_{1}, \alpha_{2}\right)$.

We claim that there exists a unique point $x_{0}^{\star}:=x_{0}^{\star}\left(y_{0}\right)$ in $\left(\alpha_{0}, \alpha_{2}\right)$ verifying $\partial q / \partial x\left(x_{0}^{\star}, y_{0}\right)=0$ (i.e., verifying that $\varphi^{\prime}\left(x_{0}^{\star}\right)=0$ ). See Fig. 11. To see the claim we consider first $y_{0} \in\left(\alpha_{0}, \alpha_{1}\right)$. From the above paragraph we know there exists $\tilde{y}_{0}$ such that $\left.\varphi\right|_{\left(\tilde{y}_{0}, \alpha_{2}\right)}$ satisfies

$$
\lim _{x \rightarrow \tilde{y}_{0}^{+}} \varphi(x)=+\infty, \quad \varphi\left(\alpha_{1}\right)=\alpha_{1} \quad \text { and } \quad \varphi\left(\alpha_{2}\right)=\alpha_{2} .
$$

Hence, since $\left.\varphi\right|_{\left(\tilde{y}_{0}, \alpha_{1}\right)}$ is smooth, we might conclude using Bolzano that there exist $x_{0}^{\star} \in\left(\alpha_{1}, \alpha_{2}\right)$ such that $\varphi^{\prime}\left(x_{0}^{\star}\right)=0$ (in fact it is a local minimum of $\varphi$ ). Uniqueness follows from the fact that there is no further change of convexity for the polynomial $p$. It remains to check the case $y_{0} \in\left(\alpha_{1}, \alpha_{2}\right)$. In this case the previous paragraph indicates the existence of $\tilde{y}_{0}$ such that $\left.\varphi\right|_{\left(\alpha_{0}, \tilde{y}_{0}\right)}$ satisfies

$$
\lim _{x \rightarrow \tilde{y}_{0}^{-}} \varphi(x)=-\infty, \quad \varphi\left(\alpha_{0}\right)=\alpha_{0} \quad \text { and } \quad \varphi\left(\alpha_{1}\right)=\alpha_{1}
$$




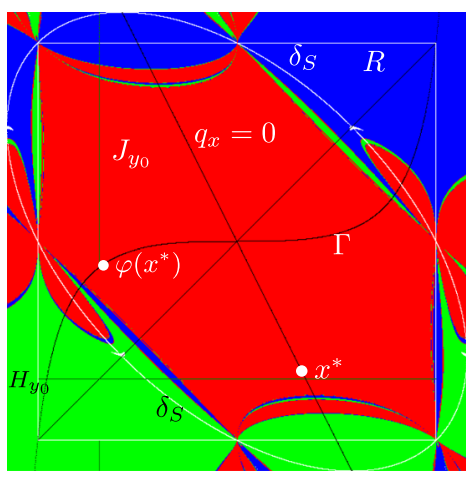

(a) Phase space of the secant map of the Chebyshev polynomial $T_{3}(x)=4 x^{3}-3 x$. Range of the picture $[-1,1] \times[-1,1]$.

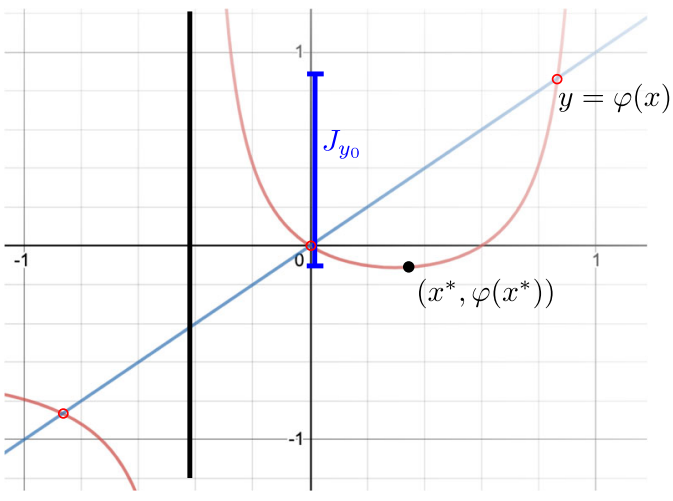

(b) Graph of $\varphi(x)=y_{0}-T_{3}\left(y_{0}\right) / q\left(x, y_{0}\right)$.

Figure 11. The secant map applied to $T_{3}$. In $\mathbf{a}$, it is shown the line $H_{y_{0}}$ with $y=y_{0}$ in the interval $\left(\alpha_{0}, \alpha_{1}\right)$. In $\mathbf{b}$, we show the graph of the related function $\varphi_{y_{0}}(x)$ when $y_{0} \in\left(\alpha_{0}, \alpha_{1}\right)$. We also plot in blue color the closed interval $J_{y_{0}}=S\left(H_{y_{0}}\right) \cap$ $\bar{R}$ contained in the segment $\overline{V_{y_{0}}}$, and introduced in Lemma 5.2 (color figure online)

Arguing similarly, we find that there exist $x_{0}^{\star} \in\left(\alpha_{0}, \alpha_{1}\right)$ such that $\varphi^{\prime}\left(x_{0}^{\star}\right)=0$ (in fact, it is a local maximum of $\varphi$ ), and uniqueness is due to the nonexistence of further convexity changes.

From the description performed so far, we clearly know how acts $S$ inside $R$, concretely fixing a value of $y_{0} \in\left(\alpha_{0}, \alpha_{2}\right)$ there exist a unique point $x_{0}^{*}:=$ $x_{0}^{*}\left(y_{0}\right) \in\left(\alpha_{0}, \alpha_{2}\right)$ solution of $\partial q / \partial x=0$ and the map $S\left(\cdot, y_{0}\right)$ is monotone for $x \in\left(\alpha_{0}, x_{0}^{*}\right)$ and monotone for $x \in\left(x_{0}^{*}, \alpha_{2}\right)$ with a turning point at $x_{0}^{*}$ and a vertical asymptote. So the lemma follows.

Next technical lemma is the last result we need to proof Theorem B. Its content gives further information about the sets

$$
\begin{aligned}
& \mathrm{LC}_{-1}:=\{(x, y) \in R \mid x \neq y, D S(x, y)=0\}, \\
& \mathrm{LC}:=\left\{S(x, y) \mid(x, y) \in \mathrm{LC}_{-1}\right\} .
\end{aligned}
$$

In particular, LC is the set of points where we cross from regions where points have either zero or two preimages in $R$. From the definition of the secant map, it is easy to see that $S(x, x)=\left(x, N_{p}(x)\right)$, where $N_{p}(x):=x-p(x) / p^{\prime}(x)$ is the Newton's map associated to $p$.

Lemma 5.3. Let $p$ be a polynomial and let $\alpha_{0}<\alpha_{1}<\alpha_{2}$ be three consecutive simple real roots of $p$. Assume that $p$ has only one inflection point, denoted by $\gamma_{0}$, in the interval $\left(\alpha_{0}, \alpha_{2}\right)$. Then, the following statements hold

(a) The set $\mathrm{LC}_{-1} \cup\left\{\left(\gamma_{0}, \gamma_{0}\right)\right\}$ is given by

$$
\Theta=\left\{(x, y) \in R \mid y \in\left(\alpha_{0}, \alpha_{2}\right), x \neq y, \quad x=x^{\star}(y)\right\} \cup\left\{\left(\gamma_{0}, \gamma_{0}\right)\right\},
$$


where $x=x^{\star}(y)$ is the unique point such that $q_{x}\left(x^{\star}(y), y\right)=0$ with $x \neq y$ unless $y=\gamma_{0}$ for which $x^{\star}\left(\gamma_{0}\right)=\gamma_{0}$. So, it can be written as the graph of a function $y \mapsto \Theta(y), y \in\left(\alpha_{0}, \alpha_{2}\right)$. Moreover $\Theta(y)$ is strictly decreasing.

(b) The set $\Gamma:=\mathrm{LC}=S(\Theta)$ is given by the graph of $N_{p}$ evaluated at the point $x^{\star}(y)$. Equivalently,

$$
\Gamma=\left\{\left(y, N_{p}\left(x^{\star}(y)\right) \mid\left(x^{*}(y), y\right) \in \Theta\right\} .\right.
$$

Let $\xi$ be such that $\Theta \cap\left\{x=\alpha_{1}\right\}=\left(\alpha_{1}, \xi\right)$, then

- If $\gamma_{0}<\alpha_{1}$ then $\Gamma$ has a local minimum at $\left(\gamma_{0}, N_{p}\left(\gamma_{0}\right)\right)$ and a local maximum at $\left(\xi, \alpha_{1}\right)$.

- If $\gamma_{0}>\alpha_{1}$ then $\Gamma$ has a local maximum at $\left(\gamma_{0}, N_{p}\left(\gamma_{0}\right)\right)$ and a local minimum at $\left(\xi, \alpha_{1}\right)$.

- If $\gamma_{0}=\alpha_{1}$ then $\Gamma$ is strictly increasing and has an inflection point at $\left(\alpha_{1}, \alpha_{1}\right)$.

(c) The points $\left(\gamma_{0}, N\left(\gamma_{0}\right)\right)$ and $\left(\xi, \alpha_{1}\right)$ belong to $\mathcal{A}^{\star}\left(\alpha_{1}\right)$.

Proof. Without lost of generality, we assume $p^{\prime}\left(\alpha_{0}\right)>0$ and so $\left.p\right|_{\left(\alpha_{0}, \alpha_{1}\right)}>0$ and $\left.p\right|_{\left(\alpha_{1}, \alpha_{2}\right)}<0$. The first part of statement (a) follows from Lemma 5.2. So, only remains to prove that $\Theta(y)$ is strictly decreasing. This fact is easy by drawing qualitatively the graph of $p$ in the interval $\left(\alpha_{0}, \alpha_{2}\right)$ under, of course, the assumption of a unique inflection point. To be more precise observe that on the one hand if $y \neq \gamma_{0}$ then $q_{x}\left(x^{\star}(y), y\right)=0$ if and only if

$$
p^{\prime}\left(x^{\star}(y)\right)=\frac{p(y)-p\left(x^{\star}(y)\right)}{y-x^{\star}(y)},
$$

and on the other hand if $y=\gamma_{0}$ then $q_{x}\left(\gamma_{0}, \gamma_{0}\right)=p^{\prime \prime}\left(\gamma_{0}\right) / 2=0$, since $\gamma_{0}$ is the unique inflection point in $\left(\alpha_{0}, \alpha_{2}\right)$. So $x^{\star}\left(\gamma_{0}\right)=\gamma_{0}$. In other words for all $y \neq \gamma_{0}$, the point $x^{\star}(y)$ is the unique point such that $x^{\star}(y) \neq y$ and its tangent line (to the graph of $p$ ) coincides with the secant line between the points $(y, p(y))$ and $\left(x^{\star}(y), p\left(x^{\star}(y)\right)\right)$ (see Fig. 12). Hence if we take a point $y \in\left(\alpha_{0}, \gamma_{0}\right)$, since $p$ is concave in this interval and convex in $\left(\gamma_{0}, \alpha_{2}\right)$, it immediately follows that $x^{\star}(y) \in\left(\gamma_{0}, \tilde{c}_{2}\right)$, where $\tilde{c_{2}} \in\left(\alpha_{1}, c_{2}\right)$ corresponds to the solution of (18) for $y=\alpha_{0}$. The tangent line at $\left(x^{*}(y), p\left(x^{*}(y)\right)\right)$ is below the graph of $p$ since $p$ is convex in the interval $\left(\gamma_{0}, \alpha_{2}\right)$. Moreover, if $y$ increases towards $\gamma_{0}$ then $x^{\star}(y)$ decreases towards $\gamma_{0}$. See Fig. 12. In the case that $y \in\left(\gamma_{0}, \alpha_{2}\right)$, the polynomial $p$ is convex in this interval with $x^{\star}(y) \in\left(\tilde{c}_{1}, \gamma_{0}\right)$ where $\tilde{c_{1}} \in\left(c_{1}, \alpha_{1}\right)$. Moreover, when $y$ decreases from $\alpha_{2}$ towards $\gamma_{0}$, then $x^{*}(y)$ increases from $\tilde{c}_{1}$ towards $\gamma_{0}$. Summarizing the closure of the curve $\Theta(y)$ is an analytic curve joining the points $\left(\alpha_{0}, \tilde{c}_{2}\right)$ and $\left(\alpha_{2}, \tilde{c}_{1}\right)$ and being decreasing on $y$.

We turn the attention to statement (b), that is the study of $\Gamma:=S(\Theta)$. Take a point $\left(x^{\star}(y), y\right)$. Its image is given by

$$
\begin{aligned}
S\left(x^{\star}(y), y\right) & =\left(y, y-\frac{p(y)}{q\left(x^{\star}(y), y\right)}\right) \\
& =\left(y, x^{\star}(y)-\frac{p\left(x^{\star}(y)\right)}{q\left(x^{\star}(y), y\right)}\right)=\left(y, N_{p}\left(x^{\star}(y)\right)\right),
\end{aligned}
$$




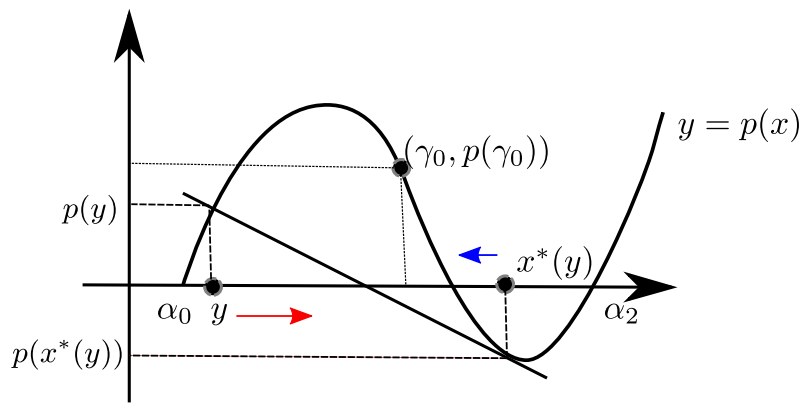

Figure 12. Sketch of the dependence of $x^{*}(y)$ with respect to $y$, when $y \in\left(\alpha_{0}, \gamma_{0}\right)$
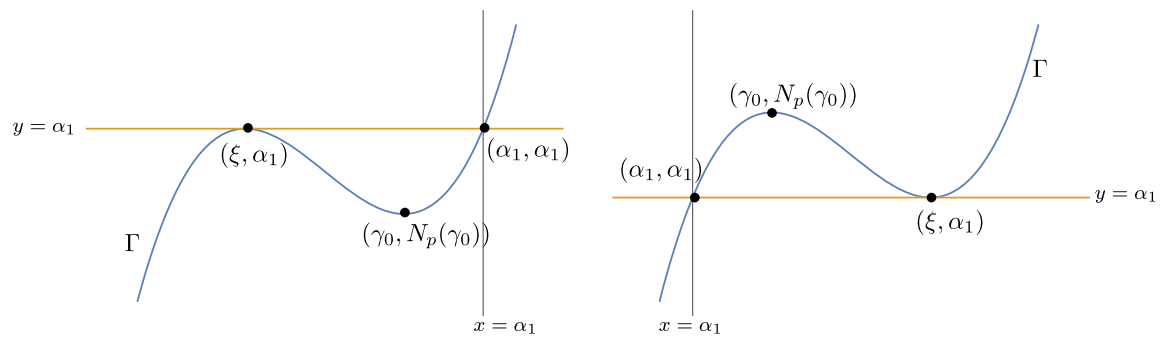

Figure 13. Qualitative draw of $\Gamma$ depending on the relative position of $\gamma_{0}$ and $\alpha_{1}$. On the left-hand side, we when $\gamma_{0}<\alpha_{1}$ and on the right hand side when $\gamma_{0}>\alpha_{1}$. The case when $\gamma_{0}=\alpha_{1}$ both local extrema collide forming an inflection point. See Fig. 11a

where the second equality follows from the general fact that the secant line passing through the points $(z, w)$ and $(w, z)$ is the same and the polynomial $q$ is symmetric. But now we can take advantage of the fact that $q\left(x^{\star}(y), y\right)=$ $p^{\prime}\left(x^{\star}(y)\right)$ since $q(x, y)$ is the slope of the secant line through $x$ and $y$ and $x^{\star}(y)$ is precisely the point where this slope coincides with the derivative (as slope) of $p$ at $x^{\star}(y)$. Thus, we conclude (17) (Fig. 13).

The second part of statement (b) follows from the computation

$$
N_{p}^{\prime}(x)=\frac{p(x) p^{\prime \prime}(x)}{\left(p^{\prime}(x)\right)^{2}},
$$

thus $x \mapsto N_{p}(x)$ exhibits two local extrema at $x=\alpha_{1}$ and $x=\gamma_{0}$ in the open interval $\left(\alpha_{0}, \alpha_{2}\right)$ since $N_{p}^{\prime}$ changes it sign at $\alpha_{1}$ and at $\gamma_{0}$. Simple computations show that if $\gamma_{0}<\alpha_{1}$ then $N_{p}$ has a local minimum at $\gamma_{0}$ and a local maximum at $\alpha_{1}$, and if $\gamma_{0}>\alpha_{1}$ then the local minimum occurs at $\alpha_{1}$ and the local maximum at $\gamma_{0}$. Now using the one-to-one relationship between $y$ and $x^{\star}(y)$, we obtain that $\Gamma$ has two local extrema at $y=\gamma_{0}$ and $y=\xi$ since $x^{\star}\left(\gamma_{0}\right)=\gamma_{0}$ and $x^{\star}(\xi)=\alpha_{1}$. If $\gamma_{0}<\alpha_{1}$ then $\Gamma$ exhibits a local maximum at $\xi$ and a local minimum at $\gamma_{0}$, if $\gamma_{0}>\alpha_{1}$ then the local maximum appears at $\gamma_{0}$ and the 
local minimum at $\xi$. Summarizing the closure of $\Gamma$ is an analytic curve joining the points $\left(\alpha_{0}, \alpha_{0}\right)$ and $\left(\alpha_{2}, \alpha_{2}\right)$ inside $R$.

It remains to show (c). We easily have that $S\left(\xi, \alpha_{1}\right)=\left(\alpha_{1}, \alpha_{1}\right)$ and we observe that $S\left(\gamma_{0}, \gamma_{0}\right)=\left(\gamma_{0}, N_{p}\left(\gamma_{0}\right)\right)$. Assume $\gamma_{0}<\alpha_{1}$ (the case $\gamma_{0}>\alpha_{1}$ follows similarly). Consider the interval $\left[\gamma_{0}, \alpha_{1}\right]$. Since $p$ is concave in this interval, we can deduce that no mater the pair of initial conditions on this interval, the secant map produces a point which is much closer to $\alpha_{1}$ than whose predecessors and always being a point in $\left[\gamma_{0}, \alpha_{1}\right]$. Hence, the whole square $\left[\gamma_{0}, \alpha_{1}\right] \times\left[\gamma_{0}, \alpha_{1}\right]$ belongs to the immediate basin.

With all these in our hands, we can start the proof of Theorem B, which is somehow a direct consequence of the previous results.

Proof of Theorem B. We reason by contradiction. Let us assume, under the assumptions, the existence of an immediate basin $\mathcal{A}^{*}\left(\alpha_{1}\right)$ not simply connected. This means that internal to the region bounded by the external frontier that we have described in Proposition 4.2, there exists at least one internal region $U$ whose points are mapped outside the immediate basin. Let us consider one point $y_{0} \in\left(\alpha_{0}, \alpha_{2}\right)$ such that the horizontal line $H_{y_{0}}$ intersects $U$. Hereafter, we can assume that $y_{0} \neq \alpha_{1}$ since, except at focal points, the rest of the points in $y=\alpha_{1}$ are in $\mathcal{A}\left(\alpha_{1}\right)$. There must be three open segments in $H_{y_{0}}$, as shown in Fig. 14, where $B \cap \mathcal{A}^{\star}\left(\alpha_{1}\right)=\emptyset$.

We first show that for the considered $y_{0} \in\left(\alpha_{0}, \alpha_{2}\right)$ the point $x^{\star}:=$ $x^{\star}\left(y_{0}\right) \in B$. Let us assume $y_{0} \in\left(\alpha_{0}, \alpha_{1}\right)$. We know that, as the value $x$ increases on $H_{y_{0}}$ over the segment $\overline{A B C}$, the shape of $\varphi_{y_{0}}(x)$ should be first decreasing and then increasing with the change of monotonicity occurring at the unique critical point $x^{\star}:=x^{\star}\left(y_{0}\right)$. Clearly, $x^{\star}$ cannot be in the segment $A$ because as $x$ increases, the image of $\varphi_{y_{0}}(x)$ must necessarily be first decreasing, then increasing, and finally decreasing again which is impossible according to Lemma 5.2. A similar argument shows that $x^{\star} \notin C$. Hence $x^{\star} \in B$, or, equivalently in $B \cap \Theta \neq \emptyset$.

We consider the image of the region $R$ by the map $S$, i.e., $S(R)$, and set $\tilde{R}=S(R) \cap R \neq \emptyset$. We know by the Lemmas 5.2 and 5.3 that the image by $S$ of each horizontal line $y=y_{0}$ for $y_{0} \in\left(\alpha_{0}, \alpha_{1}\right)$ is folded over $\Gamma$; more precisely we have that $\tilde{R}=S(R) \cap R$ is bounded below by $\Gamma$ in the $y$-interval $\left(\alpha_{0}, \alpha_{1}\right)$ and bounded above by $\Gamma$ in the $y$-interval $\left(\alpha_{1}, \alpha_{2}\right)$, as qualitatively shown in Fig. 15 (gray regions). The points in such gray region have, according to Lemma 5.1, either one or two preimages in $R$. Differently, the points belonging to the region $R \backslash \tilde{R}$ have no preimages in $R$ (white points in Fig. 15).

Let $y_{0}$ be such that the line $y=y_{0} \in\left(\alpha_{0}, \alpha_{1}\right)$ intersects the set $U$. Its image in $R$ is a segment in $x=y_{0}$ given by $\varphi_{y_{0}}(x)$, which is folded on a unique point $\varphi_{y_{0}}\left(x^{\star}\right) \in \Gamma$ (see Lemma 5.2). Moreover, the image of $\left\{y=y_{0}\right\} \cap B$ must be outside the external boundary of $\mathcal{A}^{\star}\left(\alpha_{1}\right)$. So, according to the results in Sect. 4 about the structure of the external boundary of $\mathcal{A}^{\star}\left(\alpha_{1}\right)$ (specifically Proposition 4.2), the image of $\left\{y=y_{0}\right\} \cap B$ must cross the external boundary of $\mathcal{A}^{\star}\left(\alpha_{1}\right)$ in a point of the arc $I$ connecting the focal points $Q_{0,1}$ and $Q_{1,0}$. Moreover, as we have shown in Corollary 4.3, we have that $S^{4}(I)$ is folded on a subarc $I_{1} \subset I$ whose points have two inverses, at least one, say $w$, belonging 


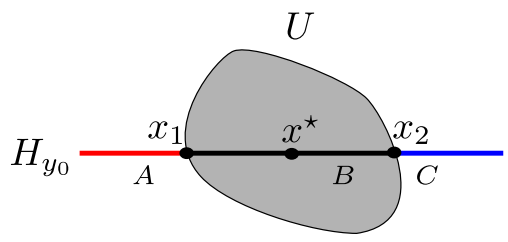

Figure 14. Sketch of the phase plane assuming that $\mathcal{A}^{*}\left(\alpha_{1}\right)$ is multiply connected

to $\Lambda \subset \partial \mathcal{A}^{\star}\left(\alpha_{1}\right)$. The border point of $I_{1}$ is a point $r_{I}=\Gamma \cap I$. Thus, the arc $I$ splits in two subarcs: $I_{1}$ (with two preimages in $R$ ) and $I_{2}=I \backslash I_{1}$ (with no preimages in $R$ ), and $r_{I}=\bar{I}_{1} \cap \bar{I}_{2}$. We claim that the described configuration of images and preimages is a contradiction with Lemma 5.1. To see the claim, we observe that there must be two points $x_{1}$ and $x_{2}$ in $\left\{y=y_{0}\right\} \cap \partial B$ mapped to $\partial \mathcal{A}^{\star}\left(\alpha_{1}\right)$ at the same point $z \in I$. But $z$ is a point in $R$ with two preimages in $R$, while the configuration implies that $z$ has three preimages in $R, x_{1}, x_{2}$ and $w$, a contradiction.

If $y_{0} \in\left(\alpha_{1}, \alpha_{2}\right)$ is such that the horizontal line $y=y_{0}$ intersects the set $U$ the arguments follows similarly. First we notice that, as before, $x^{\star}\left(y_{0}\right)$ must belong to the segment $B$ intersecting $U$. Moreover, the image of $\{y=$ $\left.y_{0}\right\} \cap B$ must be outside the external boundary of $\mathcal{A}^{\star}\left(\alpha_{1}\right)$ and the image of $\left\{y=y_{0}\right\} \cap B$ must cross the external boundary of $\mathcal{A}^{\star}\left(\alpha_{1}\right)$ in a point of the arc $K$ connecting the focal points $Q_{1,2}$ and $Q_{2,1}$. The arc $K$ splits in two subarcs $K_{1}$ and $K_{2}$ having, respectively, two (at least one, say $w^{\prime}$, belonging to $\left.J \subset \partial \mathcal{A}^{\star}\left(\alpha_{1}\right)\right)$ and none preimages in $R$. The border point of $K_{1}$ is a point $r_{K}=\Gamma \cap K=\bar{K}_{1} \cap \bar{K}_{2}$. As before, the described configuration of images and preimages is a contradiction with Lemma 5.1 since it creates a point $z^{\prime} \in K_{1}$ with three preimages.

In this case when $x$ moves in $H_{y_{0}}$ from $\alpha_{0}$ to $\alpha_{2}$, the graph of $\varphi$ exhibits two local minimum and one local maximum, and thus, the secant map could map outside $\mathcal{A}^{*}\left(\alpha_{1}\right)$ (Fig. 16).

Remark 4. From the proof of the above proposition, we conclude that a simply connected immediate basin of attraction of an internal root $\mathcal{A}^{*}\left(\alpha_{1}\right)$ is forward invariant, i.e., $S\left(\mathcal{A}^{*}\left(\alpha_{1}\right)\right) \subset \mathcal{A}^{*}\left(\alpha_{1}\right)$. This is due to the fact that no point of $\mathcal{A}^{*}\left(\alpha_{1}\right)$ can be mapped outside the set $\mathcal{A}^{*}\left(\alpha_{1}\right)$.

In Corollary 1, we collect the main results of this paper. Assuming that $p$ is a polynomial of degree $k$ with $k$ simple roots and only one inflexion point between any three consecutive roots we conclude, by Theorems A and B, that the immediate basin of attraction of an internal root is simply connected and the boundary is controlled by a 4-cycle of type I of the secant map.

We finally mention the case of the external roots, i.e., $\alpha_{0}$ and $\alpha_{n-1}$, of the polynomial $p$. In that case, a similar approach could be done as in the case of the internal ones. However, several difficulties appear. The first one is that the immediate basin of attraction of an external root is unbounded and points in the set of no definition of $\delta_{S}$ belong to this immediate basin. The second 


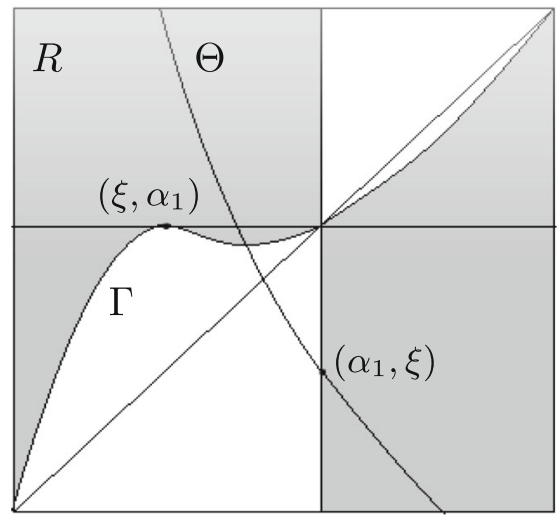

(a)

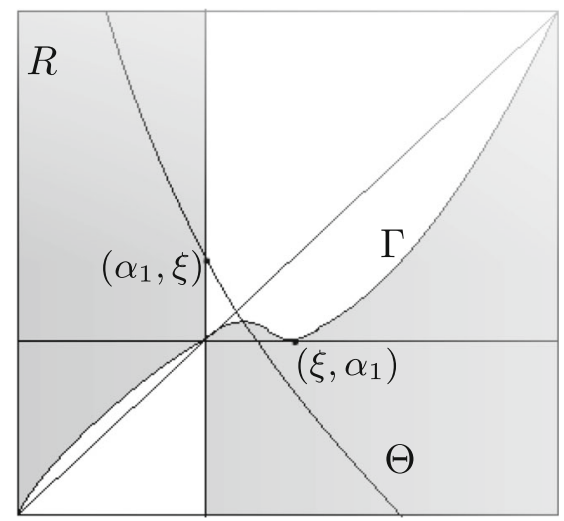

(b)

Figure 15. Qualitative picture of the image of $S(R)$ and the set $\tilde{R}=S(R) \cap R$ in gray. a Corresponds to $\gamma_{0}<\alpha_{1}$ and $\mathbf{b}$ corresponds to $\gamma_{0}>\alpha_{1}$

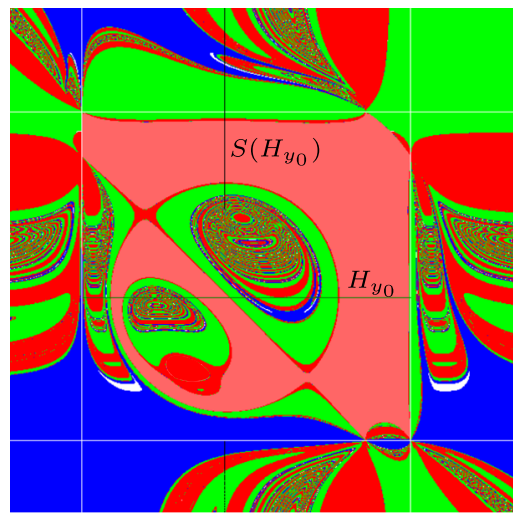

(a) Phase space of the secant map of the polynomial $p(x)=x^{5} / 5-x^{3} / 3-$ $0.05 x+0.15$. Range of the picture $[-2,2] \times[-2,2]$.

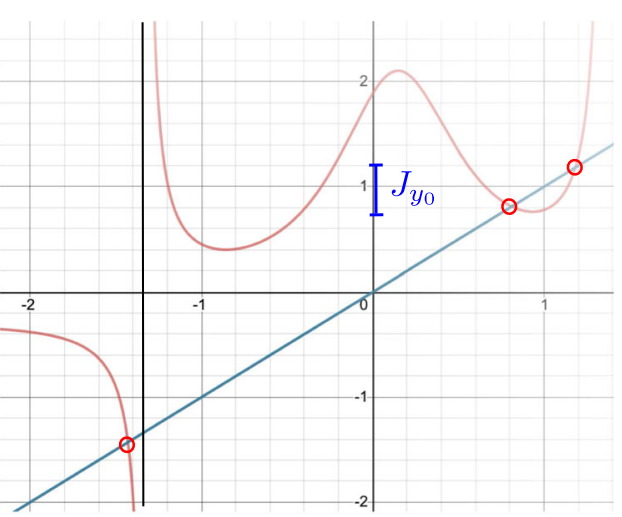

(b) Graph of $\varphi(x)=y_{0}-p\left(y_{0}\right) / q\left(x, y_{0}\right)$.

Figure 16. The secant map applied to a degree five polynomial for which the (only) internal immediate basin is multiply connected. In a, it is shown the line $H_{y_{0}}$ with $y=y_{0}$ in the interval $\left(\alpha_{0}, \alpha_{1}\right)$. In $\mathbf{b}$, we show the graph of the related function $\varphi_{y_{0}}(x)$ when $y_{0} \in\left(\alpha_{0}, \alpha_{1}\right)$. We also plot in blue color the closed interval $J_{y_{0}}=S\left(H_{y_{0}}\right) \cap \bar{R}$ contained in the segment $\overline{V_{y_{0}}}$ (see Lemma 5.2). Compare with Fig. 11 where the immediate basin is simply connected. In particular we illustrate that $\varphi$ exhibits three critical points, due to the existence of more than one change of convexity of $p$ in the relevant interval (color figure online) 
difficulty is that depending on the oddity of the degree of $p$, the boundary of $\mathcal{A}^{*}\left(\alpha_{0}\right)$ contains a critical three cycle $(c, c) \rightarrow(c, \infty) \rightarrow(\infty, c) \rightarrow(c, c)$ where $p^{\prime}(c)=0$ (degree of $p$ even) or a 4-cycle (degree of $p$ odd).

\section{Acknowledgements}

The authors would like to thank Arturo Vieiro and Armengol Gasull for helpful comments at the very initial stage of this work and the anonymous referees for valuable suggestions and for the careful reading. The second and third authors would like to thank the University of Urbino (Italy) for the kind hospitality and fruitful discussions during their research visiting period.

Funding Open Access funding provided thanks to the CRUE-CSIC agreement with Springer Nature.

Open Access. This article is licensed under a Creative Commons Attribution 4.0 International License, which permits use, sharing, adaptation, distribution and reproduction in any medium or format, as long as you give appropriate credit to the original author(s) and the source, provide a link to the Creative Commons licence, and indicate if changes were made. The images or other third party material in this article are included in the article's Creative Commons licence, unless indicated otherwise in a credit line to the material. If material is not included in the article's Creative Commons licence and your intended use is not permitted by statutory regulation or exceeds the permitted use, you will need to obtain permission directly from the copyright holder. To view a copy of this licence, visit http:// creativecommons.org/licenses/by/4.0/.

Publisher's Note Springer Nature remains neutral with regard to jurisdictional claims in published maps and institutional affiliations.

\section{References}

[1] Bedford, E., Frigge, P.: The secant method for root finding, viewed as a dynamical system. Dolomites Res. Notes Approx. 11, Special Issue Norm Levenberg, pp. 122-129 (2018)

[2] Bischi, G.-I., Gardini, L., Mira, C.: Plane maps with denominator. I. Some generic properties. Int. J. Bifurc. Chaos Appl. Sci. Eng. 9(1), 119-153 (1999)

[3] Bischi, G.-I., Gardini, L., Mira, C.: Plane maps with denominator. II. Noninvertible maps with simple focal points. Int. J. Bifurc. Chaos Appl. Sci. Eng. 13(8), 2253-2277 (2003)

[4] Bischi, G.-I., Gardini, L., Mira, C.: Plane maps with denominator. III. Nonsimple focal points and related bifurcations. Int. J. Bifurc. Chaos Appl. Sci. Eng. 15(2), 451-496 (2005)

[5] Garijo, A., Jarque, X.: Global dynamics of the real secant method. Nonlinearity 32(11), 4557-4578 (2019)

[6] Garijo, A., Jarque, X.: The secant map applied to a real polynomial with multiple roots. Discrete Contin. Dyn. Syst. 40(12), 6783-6794 (2020)

[7] Hubbard, J., Schleicher, D., Sutherland, S.: How to find all roots of complex polynomials by Newton's method. Invent. Math. 146, 1-33 (2001) 


\author{
Laura Gardini \\ Department of Economics, Society and Politics \\ University of Urbino \\ Urbino \\ Italy \\ e-mail: laura.gardini@uniurb.it

\section{Antonio Garijo} \\ Departament d'Enginyeria Informàtica i Matemàtiques \\ Universitat Rovira i Virgili \\ 43007 Tarragona \\ Catalonia \\ e-mail: antonio.garijo@urv.cat \\ Xavier Jarque \\ Departament de Matemàtiques i Informàtica at Universitat de Barelona and \\ Barcelona Graduate School of Mathematics \\ 08007 Barcelona \\ Catalonia \\ e-mail: xavier.jarque@ub.edu
}

Received: June 28, 2020.

Revised: January 4, 2021.

Accepted: August 4, 2021. 